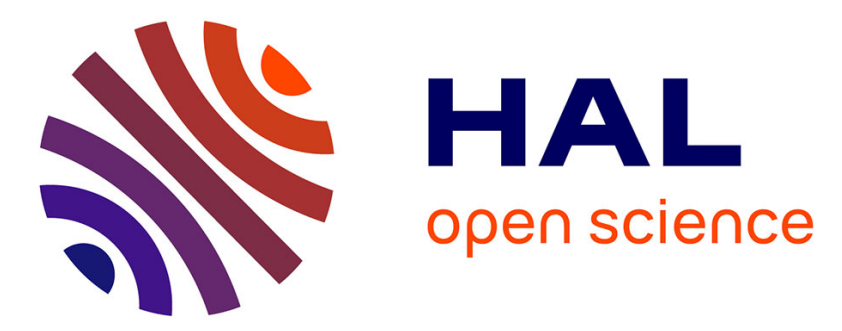

\title{
Evaluation of Shewhart time-between-events-and-amplitude control charts for correlated data
}

Dorra Rahali, Philippe Castagliola, Hassen Taleb, Michael Boon Chong Khoo

\section{- To cite this version:}

Dorra Rahali, Philippe Castagliola, Hassen Taleb, Michael Boon Chong Khoo. Evaluation of Shewhart time-between-events-and-amplitude control charts for correlated data. Quality and Reliability Engineering International, 2021, 37 (1), pp.219-241. 10.1002/qre.2731 . hal-03112645

\section{HAL Id: hal-03112645 https://hal.science/hal-03112645}

Submitted on 12 Jul 2021

HAL is a multi-disciplinary open access archive for the deposit and dissemination of scientific research documents, whether they are published or not. The documents may come from teaching and research institutions in France or abroad, or from public or private research centers.
L'archive ouverte pluridisciplinaire HAL, est destinée au dépôt et à la diffusion de documents scientifiques de niveau recherche, publiés ou non, émanant des établissements d'enseignement et de recherche français ou étrangers, des laboratoires publics ou privés. 


\title{
Evaluation of Shewhart Time-Between-Events-and-Amplitude Control Charts for Correlated Data
}

\author{
Dorra Rahali ${ }^{1,2}$, Philippe Castagliola*1, \\ Hassen Taleb ${ }^{3}$ and Michael B.C. Khoo ${ }^{4}$ \\ ${ }^{1}$ Université de Nantes \& LS2N UMR CNRS 6004, Nantes, France \\ ${ }^{2}$ Université de Tunis, Tunis, Tunisia \\ ${ }^{3}$ Institut Supérieur de Commerce et Comptabilité, University of \\ Carthage \& ARBRE Laboratory, Tunisia \\ ${ }^{4}$ School of Mathematical Sciences, Universiti Sains Malaysia, \\ Penang, Malaysia
}

July 15, 2020

Short running head: "Shewhart TBEA Control Charts for Correlated Data"

Email address of the Corresponding author: philippe. castagliola@univ-nantes.fr.

Keywords: Amplitude. Copulas. Machine breakdowns. Statistical Process

Monitoring. Time between events.

*philippe.castagliola@univ-nantes.fr (corresponding author) 


\begin{abstract}
In recent years, several techniques based on control charts have been developed for the simultaneous monitoring of the time interval $T$ and the amplitude $X$ of events, known as TBEA (Time Between Events and Amplitude) charts. However, the vast majority of the existing works have some limitations. Firstly, they usually focus on statistics based on the ratio $\frac{X}{T}$ and, secondly, they only investigate a reduced number of potential distributions, i.e. the exponential distribution for $T$ and the Normal distribution for $X$. Moreover, until now, very few research papers have considered the potential dependence between $T$ and $X$. In this paper, we investigate three different statistics, denoted as $Z_{1}, Z_{2}$ and $Z_{3}$, for monitoring TBEA data in the case of three potential distributions (Gamma, Normal and Weibull), for both $T$ and $X$, using Copulas as a mechanism to model the dependence. An illustrative example considering times between machine breakdowns and associated maintenance illustrates the use of TBEA control charts.
\end{abstract}

Keywords: Amplitude. Copulas. Machine breakdowns. Statistical Process Monitoring. Time between events.

\title{
1 Introduction
}

Control charts are one of the most effective techniques in Statistical Process Monitoring (SPM) to monitor manufacturing processes or critical events E such as the ones occuring in healthcare or in geological (earthquakes or volcanic eruptions) applications. The definition of an event E clearly depends on the situation considered. For instance, in the manufacturing industry, it means the occurrence of a nonconforming product; in reliability engineering, it means the failure of a specific system, etc. (see $\mathrm{Wu}$ et al. ${ }^{1}$ ). In general, a critical event is defined by two important characteristics: i) the time interval $T$ measured by the duration between two successive occurrences of the event $E$ and, ii) its amplitude (cost, number of casualties, ...) $X$ which reflects the correspending effect, see Cheng et al. ${ }^{2}$.

The problem of monitoring Time Between Events was introduced for the first time by Calvin ${ }^{3}$. Lucas $^{4}$ and Vardeman and Ray ${ }^{5}$ extended this work by studying new control charts for the monitoring of Time Between Events (TBE). From that moment, many TBE control charts have been developed. The TBE exponential chart has been studied by Chan et al. ${ }^{6}$ and Xie et al. ${ }^{7}$. Bourke ${ }^{8}$ developed a geometric CUSUM chart for monitoring TBE data. Gan ${ }^{9}$, Borror et al. ${ }^{10}$ and Shafae et al. ${ }^{11}$ investigated an exponential TBE cumulative sum (CUSUM) control chart. An exponentially weighted moving average (EWMA) control chart for monitoring the rate of occurrences of rare events has been developped by Gan ${ }^{12}$. Liu et al. ${ }^{13}$ discussed the performance of continuous TBE charts among the CQC, CQC-r, exponential EWMA and exponential CUSUM charts. Zhang et al. ${ }^{14}$ investigated a Gamma chart to monitor the TBE and they developed a new method based on a random-shift model for calculating the out-of-control ATS. Xie et al. ${ }^{15}$ discussed the application of TBE control charts for health management. Recently Fang et al. ${ }^{16}$ proposed a generalized group runs TBE chart for a homogenous Poisson failure process. 
As the effect of an event $\mathrm{E}$ is not only quantified by its frequency $T$ but also by its amplitude $X$, a combined scheme for monitoring the time interval using a $T$ chart and the amplitude using a $X$ chart have been introduced by Wu et al. ${ }^{17}$. The corresponding control chart is called a Time Between Events and Amplitude (TBEA) chart. After the paper of Wu et al. ${ }^{17}$, several single TBEA charts have been developed (see $\mathrm{Wu}$ and $\mathrm{Qu}{ }^{18}$, Qu et al. ${ }^{19}$, Qu et al. ${ }^{20}$ ). Very recently Ali and Pievatolo ${ }^{21}$ introduced a new control chart based on the assumption of a renewal process with rewards, where the reward represents the magnitude, and a magnitude-over-threshold condition represents the occurrence of an event.

All the TBEA control charts presented above assume that the time between events $T$ and their amplitudes $X$ are independent variables. However, in practice, they may not necessarily be independent. More specifically, there are many situations where it seems expectable (and logical) that when the time between events becomes shorter, then the corresponding amplitude becomes larger. For instance, small amplitude earthquakes may occur with a low frequency (large time between events) and, suddenly, the frequency of the occurrence of these earthquakes may increase (shorter time between events) with a correlated increase in their amplitudes. As another example, some forest fires may occur with a low frequency and small amplitude (surface burned) during the "humid season" and, when the "dry season" comes, the time between the occurrence of forest fires tends to become shorter and their amplitude tends to increase (see the 2019 forest fires in Amazonia or Siberia). As the illustrative example will show, the contrary may also happen, i.e. time between events becomes shorter and amplitude becomes smaller. Whatever the situation, it seems important to investigate the dependency between time between events $T$ and their amplitudes $X$. Until now, very few research papers have investigated TBEA control charts by considering the potential dependence between the two variables. Cheng and Mukherjee ${ }^{22}$ were the first to investigate a TBEA control chart by taking into account the dependence between the frequency and the amplitude using a $T^{2}$ chart based on transformed data. They have used a Smith-Adelfang-Tubbs (SAT) bivariate Gamma distribution to model the joint probability of $T$ and $X$ and they have shown that the proposed chart is more effecient when the shift size is moderate to large. Then, Cheng et al. ${ }^{2}$ developed a multivariate exponentially weighted moving average (MEWMA) scheme also based on a bivariate Gamma distribution, to jointly monitor the frequency and the amplitude of an event. A comparison is conducted between three kinds of charts under eight shift domains and the results show that the proposed MEWMA procedure outperforms other charts in most scenarios. Very recently, Sanusi et al. ${ }^{23}$ proposed a Max-EWMA type chart using the maximum of the absolute values of two EWMA statistics, one for controlling the magnitude and the other for the frequency of an event. The limitation of those studies is that they only considered the bivariate Gamma distribution to model the joint probability of $T$ and $X$, while in this paper, we propose a general mechanism based on Copulas that allows a very flexible choice for the distribution of $T$ and $X$.

In order to model the dependence between the time $T$ and the amplitude $X$, in this paper we suggest the use of Copulas (popularized by Sklar ${ }^{24}$ ) which allow the degree of association between these variables to be quantified without having to actually assume anything concerning their distributions. For instance, 
Copulas have been used in the financial domain. Several researchers also used Copulas for defining and implementing control charts. Fatahi et al. ${ }^{25}$ proposed a bivariate control chart based on Copulas. A Copula Markov CUSUM chart for monitoring bivariate auto-correlated data has been developed by Dokouhaki and Noorossana ${ }^{26}$. Busababodhin and Amphanthong ${ }^{27}$ studied various types of Copulas modeling for several multivariate control charts. Very recently, Sukparungsee et al. ${ }^{28}$ proposed five different types of Copulas for the Hotelling's $T^{2}$ control chart and they evaluated the statistical measures of performance using Monte Carlo simulations.

In this paper we propose to extend the preliminary work in Rahali et al. ${ }^{29}$ and use three kinds of Copulas in order to investigate the properties of one-sided Shewhart TBEA charts for three different statistics $Z_{1}, Z_{2}$ and $Z_{3}$ which all depend on $T$ (time) and $X$ (amplitude). The main limitations of this contribution are i) the fact that a parametric distribution has to be chosen for $X$ and $T$ (without being exhaustive, this paper nevertheless investigates three distributions: Gamma, Normal and Weibull), ii) the parameters of these distributions, as well as the Copulas dependence parameter have to be known or, at least, accurately estimated.

Finally, it is important to note that the kind of events $\mathrm{E}$ we are interested in are different from the so-called "recurrence data" that can be found in survival analysis for which some right-censoring is likely to occur (i.e. at time $t$, the interarrival time between the $N(t)$ th event and the $(N(t)+1)$ th event is unobserved and we only know that it is longer than $t-S_{N(t)}$, where $S_{k}$ is the occurrence time of the $k$ th event), see for intance, Wang et al. ${ }^{30}$, Huang and Wang ${ }^{31}$ and Rondeau ${ }^{32}$. For example, in the case of cancer patients who have already been treated, the time-between-events is the time between two cancer recurrences. In this situation, right-censoring may (unfortunately) happen as, for some patients of the sample, after several recurrences of the disease, the last time-between-events is missing because they passed away (they never recovered from the cancer or they died because of some other reasons). Moreover, recurrence data in survival analysis, usually involve a sample (group of patients with the same disease, for instance) while, in our case, we only focus on a single phenomena. In conclusion, in our case, right-censoring is not supposed to happen because the kind of event $\mathrm{E}$ we are interested in (earthquakes, tsunamis, fires and, even, machine breakdown occurences in our illustrative example) assumes that i) no matter what the situation is, this event $\mathrm{E}$ will necessarily re-happen "naturally" in the future, ii) it costs nothing to wait for the next occurence of this event, iii) there is no sample to deal with.

The paper is structured as follows: in section 2 we introduce the dependence structure between $T$ and $X$ using Copulas, we define the statistics $Z_{1}, Z_{2}$ and $Z_{3}$ and derive their cumulative distribution functions, we define the upper control limits of the corresponding TBEA charts and derive their time to signal properties. Then, in Section 3, we investigate the EATS properties of the three TBEA charts for 8 distributional scenarios, 3 different Copulas and several levels of correlation. Finally, in Section 4, we propose an illustrative example involving the time between consecutive machine breakdowns and their associate maintenance costs. Conclusions with comments and recommendations for future research are 
given in Section 5 .

\section{TBEA charts}

Let $T$ be the time interval between two consecutive occurrences of a specific event $\mathrm{E}$ and let $X$ be the corresponding magnitude of this event. In this paper, we assume that $(X, T) \in \mathbb{R}_{+}^{2}$ and their joint continuous c.d.f. (cumulative distribution function) is

$$
F_{(T, X)}(t, x)=C\left(F_{T}(t), F_{X}(x) \mid \theta\right),
$$

where $F_{T}(t)$ and $F_{X}(x)$ are the marginal c.d.f. of $T$ and $X$, respectively, $C(u, v \mid \theta)$ is a Copula containing all information on the dependence structure between $T$ and $X$ while $\theta$ is a dependence parameter that measures the dependence between the marginals. Let

$$
f_{(T, X)}(t, x)=c\left(F_{T}(t), F_{X}(x) \mid \theta\right) f_{T}(t) f_{X}(x)
$$

be the joint p.d.f. (probability distribution function) of $(X, T)$ where $f_{T}(t)$ and $f_{X}(x)$ are the marginal p.d.f.'s of $T$ and $X$, respectively, and $c(u, v \mid \theta)=\frac{\partial C(u, v \mid \theta)}{\partial u \partial v}$ is the Copula density.

Let $\mu_{T}>0$ and $\mu_{X}>0$ be the (marginal) means of $T$ and $X$, respectively. By definition, when the process is in-control, we have $\mu_{T}=\mu_{T_{0}}, \mu_{X}=\mu_{X_{0}}$ and, when the process is out-of-control, we have $\mu_{T}=\mu_{T_{1}}, \mu_{X}=\mu_{X_{1}}$. In order to not favor one random variable over the other one (their scale can be very different), the new random variables $T^{\prime}$ and $X^{\prime}$ are introduced as the in-control standardized counterparts of $T$ and $X$, i.e.

$$
\begin{aligned}
& T^{\prime}=\frac{T}{\mu_{T_{0}}}, \\
& X^{\prime}=\frac{X}{\mu_{X_{0}}} .
\end{aligned}
$$

By definition, when the process is in-control, $E\left(T^{\prime}\right)=E\left(X^{\prime}\right)=1$. The marginal c.d.f. of $T^{\prime}$ and $X^{\prime}$ are $F_{T^{\prime}}(t)=F_{T}\left(t \mu_{T_{0}}\right)$ and $F_{X^{\prime}}(x)=F_{X}\left(x \mu_{X_{0}}\right)$. Let $F_{\left(T^{\prime}, X^{\prime}\right)}(t, x)$ and $f_{\left(T^{\prime}, X^{\prime}\right)}(t, x)$ be the joint c.d.f. and p.d.f. of $\left(X^{\prime}, T^{\prime}\right) \in \mathbb{R}_{+}^{2}$, respectively. It is easy to prove that

$$
\begin{gathered}
F_{\left(T^{\prime}, X^{\prime}\right)}(t, x)=C\left(F_{T}\left(t \mu_{T_{0}}\right), F_{X}\left(x \mu_{X_{0}}\right) \mid \theta\right), \\
f_{\left(T^{\prime}, X^{\prime}\right)}(t, x)=\mu_{T_{0}} \mu_{X_{0}} c\left(F_{T}\left(t \mu_{T_{0}}\right), F_{X}\left(x \mu_{X_{0}}\right) \mid \theta\right) f_{T}\left(t \mu_{T_{0}}\right) f_{X}\left(x \mu_{X_{0}}\right) .
\end{gathered}
$$

As in Rahali et al. ${ }^{29}$, in order to simultaneously monitor the time between an event $\mathrm{E}$ and its amplitude, we investigate three different statistics $Z=$ $Z\left(T^{\prime}, X^{\prime}\right)$, denoted as $Z_{1}, Z_{2}$ and $Z_{3}$, all functions of $T^{\prime}$ and $X^{\prime}$, having the following properties: i) $Z \uparrow$ if either $T^{\prime} \downarrow$ or $X^{\prime} \uparrow$, ii) $Z \downarrow$ if either $T^{\prime} \uparrow$ or $X^{\prime} \downarrow$.

A first possible choice for the statistic $Z$ (denoted as the $Z_{1}$ statistic) is

$$
Z_{1}=X^{\prime}-T^{\prime}
$$

If we integrate over all the couples $\left(X^{\prime}, T^{\prime}\right) \in \mathbb{R}_{+}^{2}$ satisfying $Z_{1}=X^{\prime}-T^{\prime} \leq z$, then the c.d.f. $F_{Z_{1}}(z)$ of $Z_{1}$ is equal to 
- if $z<0$ (see Figure 1 (a), where the grey area represents the region satisfying $\left.Z_{1}=X^{\prime}-T^{\prime} \leq z<0\right)$

$$
F_{Z_{1}}(z)=\int_{0}^{\infty} \int_{x-z}^{\infty} f_{\left(T^{\prime}, X^{\prime}\right)}(t, x) \mathrm{d} t \mathrm{~d} x
$$

- if $z>0$ (see Figure 1 (b), where the grey area represents the region satisfying $\left.Z_{1}=X^{\prime}-T^{\prime} \leq z>0\right)$

$$
\begin{aligned}
F_{Z_{1}}(z) & =\int_{0}^{z} \int_{0}^{\infty} f_{\left(T^{\prime}, X^{\prime}\right)}(t, x) \mathrm{d} t \mathrm{~d} x+\int_{z}^{\infty} \int_{x-z}^{\infty} f_{\left(T^{\prime}, X^{\prime}\right)}(t, x) \mathrm{d} t \mathrm{~d} x \\
& =F_{X^{\prime}}(z)+\int_{z}^{\infty} \int_{x-z}^{\infty} f_{\left(T^{\prime}, X^{\prime}\right)}(t, x) \mathrm{d} t \mathrm{~d} x
\end{aligned}
$$

Since $F_{X^{\prime}}(z)=0$ when $z<0$ and $f_{\left(T^{\prime}, X^{\prime}\right)}(t, x)=0$ when either $t<0$ or $x<0$, the last result can be generalized to $z \in \mathbb{R}$.

A second possible choice for the statistic $Z$ (denoted as the $Z_{2}$ statistic) is

$$
Z_{2}=\frac{X^{\prime}}{T^{\prime}}
$$

The random variable $Z_{2}$ is defined on $[0,+\infty)$ and its c.d.f. $F_{Z_{2}}(z)$ can be obtained by integrating over all the couples $\left(X^{\prime}, T^{\prime}\right) \in \mathbb{R}_{+}^{2}$ satisfying $Z_{2}=$ $\frac{X^{\prime}}{T^{\prime}} \leq z$ (see Figure 1 (c), where the grey area represents the region satisfying $\left.Z_{2}=\frac{X^{\prime}}{T^{\prime}} \leq z\right)$, i.e.

$$
F_{Z_{2}}(z)=\int_{0}^{\infty} \int_{\frac{x}{z}}^{\infty} f_{\left(T^{\prime}, X^{\prime}\right)}(t, x) \mathrm{d} t \mathrm{~d} x .
$$

Finally, a possible third choice for the statistic $Z$ (denoted as the $Z_{3}$ statistic) is

$$
Z_{3}=X^{\prime}+\frac{1}{T^{\prime}}
$$

This statistic can be considered as a "hybrid" of the two previous ones. The random variable $Z_{3}$ is also defined on $[0,+\infty)$ and its c.d.f. $F_{Z_{3}}(z)$ can be obtained by integrating over all the couples $\left(X^{\prime}, T^{\prime}\right) \in \mathbb{R}_{+}^{2}$ satisfying $Z_{3}=$ $X^{\prime}+\frac{1}{T^{\prime}} \leq z$ (see Figure $1(\mathrm{~d})$, where the grey area represents the region satisfying $\left.Z_{3}=X^{\prime}+\frac{1}{T^{\prime}} \leq z\right)$, i.e.

$$
F_{Z_{3}}(z)=\int_{0}^{z} \int_{\frac{1}{z-x}}^{\infty} f_{\left(T^{\prime}, X^{\prime}\right)}(t, x) \mathrm{d} t \mathrm{~d} x .
$$

The control limits $\mathrm{LCL}_{Z}$ and $\mathrm{UCL}_{Z}$ of the TBEA (Time Between Event and Amplitude) charts based on the statistic $Z \in\left\{Z_{1}, Z_{2}, Z_{3}\right\}$ defined in equations (7), (10) and (12) are equal to

$$
\begin{aligned}
\mathrm{LCL}_{Z} & =F_{Z}^{-1}\left(\alpha_{L} \mid \mu_{T_{0}}, \mu_{X_{0}}\right) \\
\mathrm{UCL}_{Z} & =F_{Z}^{-1}\left(1-\alpha_{U} \mid \mu_{T_{0}}, \mu_{X_{0}}\right),
\end{aligned}
$$




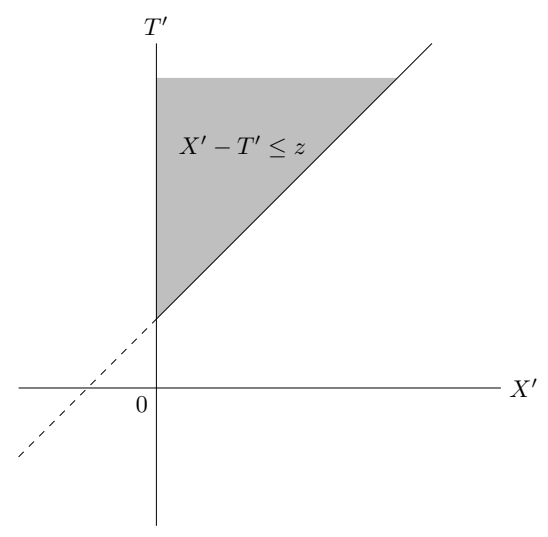

(a) Region satisfying $Z_{1}=X^{\prime}-T^{\prime} \leq z<0$

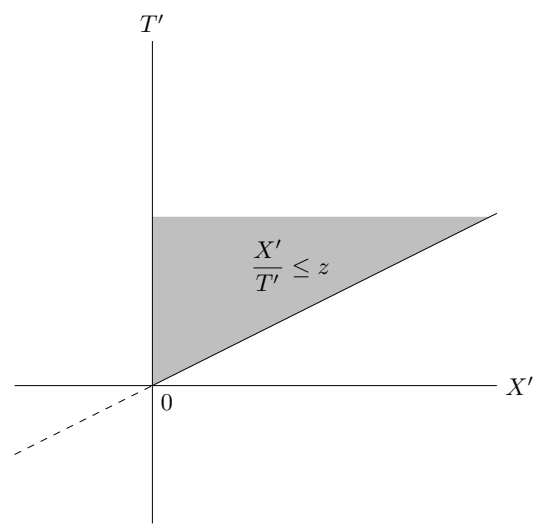

(c) Region satisfying $Z_{2}=\frac{X^{\prime}}{T^{\prime}} \leq z$

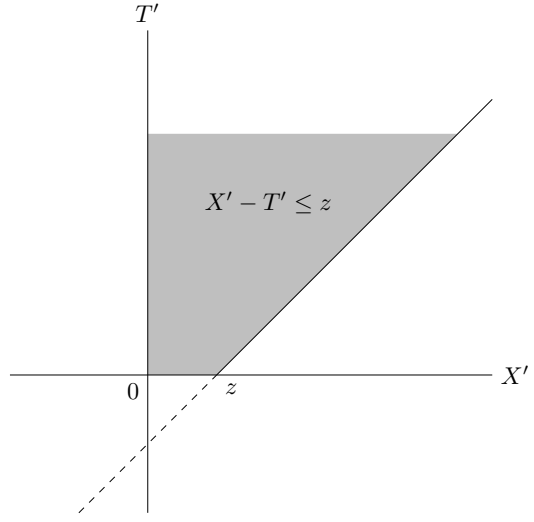

(b) Region satisfying $Z_{1}=X^{\prime}-T^{\prime} \leq z>0$

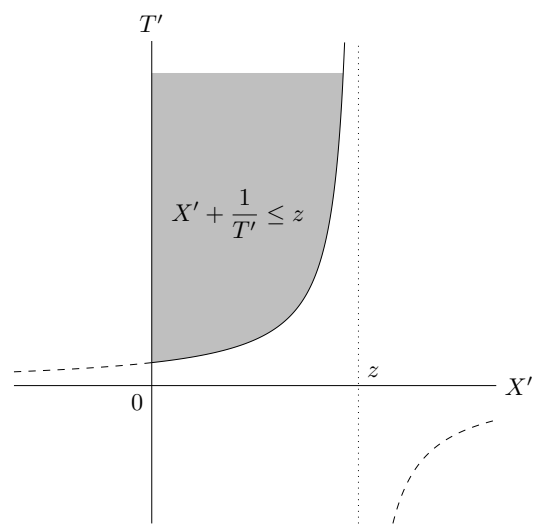

(d) Region satisfying $Z_{3}=X^{\prime}+\frac{1}{T^{\prime}} \leq z$

Figure 1: Integration areas used for statistics $Z_{1}=X^{\prime}-T^{\prime} \leq z$ ((a) and (b)), $Z_{2}=\frac{X^{\prime}}{T^{\prime}} \leq z(\mathrm{c})$ and $Z_{3}=X^{\prime}+\frac{1}{T^{\prime}} \leq z(\mathrm{~d})$. 
where $F_{Z}^{-1}\left(\ldots \mid \mu_{T_{0}}, \mu_{X_{0}}\right)$ is the inverse c.d.f. of $Z, \alpha_{L}=\xi \alpha$ and $\alpha_{U}=(1-\xi) \alpha$ are the lower-sided and upper-sided components of the Type I error $\alpha$, respectively, with $\xi$ being the detection power allocation factor defined as $\xi=\frac{\alpha_{L}}{\alpha_{L}+\alpha_{U}}$. Since it is important to consider the case of upward shift on $Z$, we will assume that $\xi=0$ and, consequently, we only have a single upper-sided control limit $\mathrm{UCL}_{Z}$.

A performance characteristic for the TBEA charts is the the average time to signal (ATS). The out-of-control ATS indicates the average time required to signal an out-of control case, whereas the in-control $\mathrm{ATS}_{0}$ is used as a measure of the false alarm rate. The Type II error $\beta$ of the TBEA charts based on the statistic $Z \in\left\{Z_{1}, Z_{2}, Z_{3}\right\}$ is equal to

$$
\beta=F_{Z}\left(\mathrm{UCL}_{Z} \mid \mu_{T}, \mu_{X}\right)-F_{Z}\left(\mathrm{LCL}_{Z} \mid \mu_{T}, \mu_{X}\right) .
$$

As the TBEA chart is a Shewhart type chart, the average run length, ARL = $E(\mathrm{RL})=\frac{1}{1-\beta}$. Let $\ell \in\{1,2,3, \cdots\}$ and $T_{1}, T_{2}, T_{3}, \ldots$ be the time between two consecutive events. The Time to Signal, TS is equal to

$$
\mathrm{TS}=\sum_{\ell=1}^{\mathrm{RL}} T_{\ell} .
$$

The Average Time to Signal and the Standard-Deviation of the Time to Signal are calculated by

$$
\begin{aligned}
& \begin{aligned}
& \mathrm{ATS}=E(\mathrm{TS})=E(T) E(\mathrm{RL})=\mu_{T} \times \mathrm{ARL}=\frac{\mu_{T}}{1-\beta}, \\
& \mathrm{SDTS}=\sigma(\mathrm{TS})=\sqrt{V(T) E(\mathrm{RL})+E^{2}(T) V(\mathrm{RL})}, \\
&=\sqrt{\sigma_{T}^{2} \times \mathrm{ARL}+\mu_{T}^{2} \times \mathrm{SDRL}^{2}}, \\
&=\sqrt{\frac{\sigma_{T}^{2}}{1-\beta}+\frac{\mu_{T}^{2} \beta}{(1-\beta)^{2}}} .
\end{aligned}
\end{aligned}
$$

When the process is in-control, we have $1-\beta=\alpha$ and, consequently

$$
\mathrm{ATS}_{0}=\frac{\mu_{T_{0}}}{\alpha} \Leftrightarrow \alpha=\frac{\mu_{T_{0}}}{\mathrm{ATS}_{0}} .
$$

\section{$3 \quad$ Numerical analysis}

In this section, we consider three types of distribution for $T$ and $X$ that depend on two parameters, $a$ and $b$. We denote $a_{0}$ and $b_{0}$ as the in-control values of these parameters, respectively.

- The Gamma distribution has parameters $a>0, b>0$ and p.d.f.

$$
f_{G}(x \mid a, b)=\frac{\exp \left(-\frac{x}{b}\right) x^{a-1}}{b^{a} \Gamma(a)} .
$$


- The Normal distribution has parameters $a, b>0$ as in Rahali et al. ${ }^{29}$, instead of the conventional notation $(\mu, \sigma)$ and p.d.f. $f_{N}(x \mid a, b)$.

- The Weibull distribution has parameters $a>0, b>0$ and p.d.f.

$$
f_{W}(x \mid a, b)=\frac{a}{b}\left(\frac{x}{b}\right)^{a-1} \exp \left(-\left(\frac{x}{b}\right)^{a}\right) \text {. }
$$

To be more specific, in this paper, we have chosen to investigate

- the Gamma, Normal and Weibull distributions for the random variable $X$ (amplitude).

- only the Gamma and the Weibull distributions for the random variable $T$ (time). The Normal distribution is actually not the most suitable distribution for time data and we have decided to not investigate it.

In order to fairly compare the three TBEA charts defined in Section 2, we considered 8 in-control situations. Table 1 shows the in-control parameters $a_{0}$ and $b_{0}$ and the skewness coefficient $\gamma_{0}$ corresponding to an in-control mean $\mu_{0}=10$ and an in-control standard-deviation $\sigma_{0} \in\{1,2,5\}$ (for the Normal distribution we only consider $\left.\sigma_{0} \in\{1,2\}\right)$. The formulae from which we obtain the values of $a_{0}$ and $b_{0}$ from $\mu_{0}$ and $\sigma_{0}$ are given below:

- For the Gamma distribution:

$$
a_{0}=\left(\frac{\mu_{0}}{\sigma_{0}}\right)^{2}, \quad b_{0}=\frac{\mu_{0}}{a_{0}} .
$$

- For the Normal distribution:

$$
a_{0}=\mu_{0}, \quad b_{0}=\sigma_{0} .
$$

- For the Weibull distribution: $a_{0}$ is the solution of the equation (obtained using a univariate root finder)

$$
\frac{\Gamma\left(\frac{2}{a_{0}}+1\right)}{\Gamma^{2}\left(\frac{1}{a_{0}}+1\right)}=\left(\frac{\sigma_{0}}{\mu_{0}}\right)^{2}+1,
$$

and

$$
b_{0}=\frac{\mu_{0}}{\Gamma\left(\frac{1}{a_{0}}+1\right)} .
$$

For the sake of clarity, the distributions corresponding to these 8 cases (i.e. Gamma with $\mu_{0}=10$ and $\sigma_{0} \in\{1,2,5\}$, Normal with $\mu_{0}=10$ and $\sigma_{0} \in\{1,2\}$ and Weibull with $\mu_{0}=10$ and $\left.\sigma_{0} \in\{1,2,5\}\right)$ are displayed in Figure 2.

In this paper, we have chosen to investigate 3 types of bivariate Archimedean Copula: 
Table 1: Distributions for $T$ and $X$ considered in this paper.

\begin{tabular}{cccccc}
\hline Distribution & $a_{0}$ & $b_{0}$ & $\mu_{0}$ & $\sigma_{0}$ & $\gamma_{0}$ \\
\hline Gamma & 100 & 0.1 & 10 & 1 & 0.2 \\
& 25 & 0.4 & 10 & 2 & 0.4 \\
Normal & 4 & 2.5 & 10 & 5 & 1 \\
& 10 & 1 & 10 & 1 & 0 \\
Weibull & 10 & 2 & 10 & 2 & 0 \\
& 12.1534 & 10.4304 & 10 & 1 & -0.7155 \\
& 5.7974 & 10.7998 & 10 & 2 & -0.3519 \\
& 2.1013 & 11.2906 & 10 & 5 & 0.5664 \\
\hline
\end{tabular}

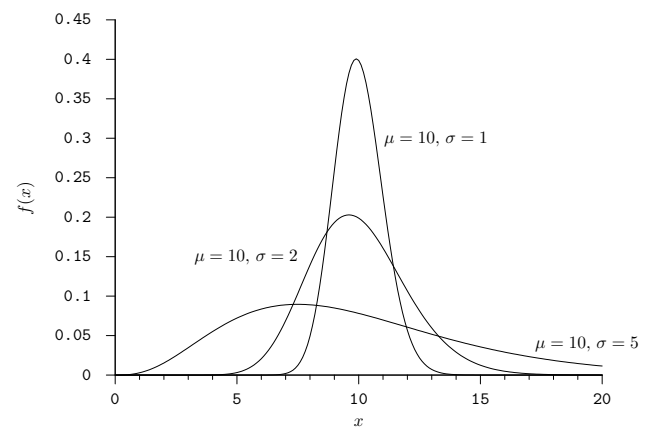

(a) Gamma with $\mu_{0}=10$ and $\sigma_{0} \in\{1,2,5\}$

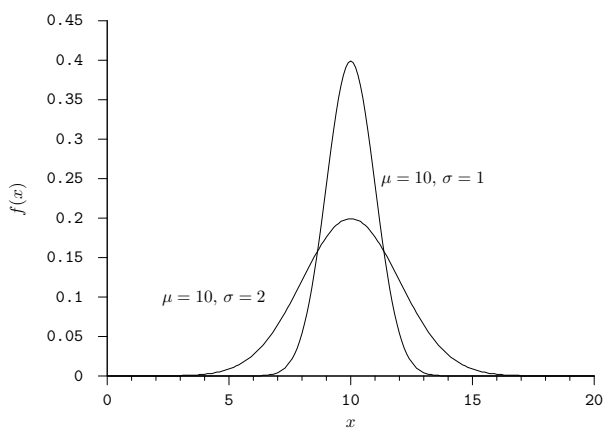

(b) Normal with $\mu_{0}=10$ and $\sigma_{0} \in\{1,2\}$

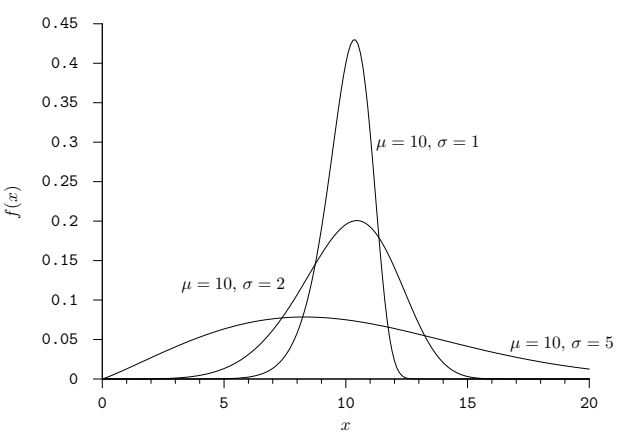

(c) Weibull with $\mu_{0}=10$ and $\sigma_{0} \in\{1,2,5\}$

Figure 2: Distributions considered in this paper (a) Gamma with $\mu_{0}=10$ and $\sigma_{0} \in\{1,2,5\}$, (b) Normal with $\mu_{0}=10$ and $\sigma_{0} \in\{1,2\}$ and (c) Weibull with $\mu_{0}=10$ and $\sigma_{0} \in\{1,2,5\}$. 
- The Frank ${ }^{33}$ Copula defined as

$$
C(u, v \mid \theta)=-\frac{1}{\theta} \ln \left(1+\frac{\left(e^{-\theta u}-1\right)\left(e^{-\theta v}-1\right)}{e^{-\theta}-1}\right),
$$

where $\theta \in \mathbb{R} \backslash\{0\}$. This Copula is symmetric and can be used to model dependence structures with either positive or negative correlation. For the Frank Copula, it can be proven that the Kendall's rank correlation coefficient $\tau$ is related to the dependence parameter $\theta$ through the following equation

$$
\tau=1+\frac{4\left(D_{1}(\theta)-1\right)}{\theta},
$$

where $D_{1}(\theta)$ is the Debye function of the first kind defined as

$$
D_{1}(\theta)=\frac{1}{\theta} \int_{0}^{\theta} \frac{t}{e^{t}-1} \mathrm{~d} t .
$$

By solving (27) for $\theta$, we can obtain the value of $\theta$ in the function of $\tau$.

- The Clayton ${ }^{34}$ Copula defined as

$$
C(u, v \mid \theta)=\max \left(0, u^{-\theta}+v^{-\theta}-1\right)^{-\frac{1}{\theta}},
$$

where $\theta \in[-1, \infty) \backslash\{0\}$. This is an asymmetric Copula exhibiting a larger dependence in the negative tail than in the positive one. For the Clayton Copula, it can be proved that the Kendall's rank correlation coefficient $\tau$ is related to the dependence parameter $\theta$ through the following equation

$$
\tau=\frac{\theta}{\theta+2} \Leftrightarrow \theta=\frac{2 \tau}{1-\tau} .
$$

- The Gumbel ${ }^{35}$ (a.k.a. Gumbel-Hougard) Copula is defined as

$$
C(u, v \mid \theta)=\exp \left(-\left((-\ln (u))^{\theta}+(-\ln (v))^{\theta}\right)^{\frac{1}{\theta}}\right),
$$

where $\theta \in[1, \infty)$. This is also an asymmetric Copula exhibiting a larger dependence in the positive tail than in the negative one. For the Gumbel Copula, it can be proved that the Kendall's rank correlation coefficient $\tau$ is related to the dependence parameter $\theta$ through the following equation

$$
\tau=1-\frac{1}{\theta} \Leftrightarrow \theta=\frac{1}{1-\tau} .
$$

In order to facilitate the use of the Frank, Clayton and Gumbel Copulas, Table 2 simply provides pre-computed values of $\theta$ for several selected values of the Kendall's rank correlation coefficient $\tau \in\{0,0.1,0.2, \ldots, 0.9\}$.

The upper control limits $\mathrm{UCL}_{Z_{1}}, \mathrm{UCL}_{Z_{2}}$ and $\mathrm{UCL}_{Z_{3}}$ of the 3 TBEA charts based on the statistic $Z \in\left\{Z_{1}, Z_{2}, Z_{3}\right\}$ have been computed (and can be found in Tables 3, 4 and 5 for the Frank, Clayton and Gumbel Copulas, respectively) for 
Table 2: Pre-computed values of $\theta$ for several selected values of $\tau \in$ $\{0,0.1,0.2, \ldots, 0.9\}$

\begin{tabular}{cccc}
\cline { 2 - 4 }$\tau$ & \multicolumn{3}{c}{$\theta$} \\
\hline 0.0 & 0.00 & 0.00 & 1.00 \\
0.1 & 0.91 & 0.22 & 1.11 \\
0.2 & 1.86 & 0.50 & 1.25 \\
0.3 & 2.92 & 0.86 & 1.43 \\
0.4 & 4.16 & 1.33 & 1.67 \\
0.5 & 5.74 & 2.00 & 2.00 \\
0.6 & 7.93 & 3.00 & 2.50 \\
0.7 & 11.41 & 4.67 & 3.33 \\
0.8 & 18.19 & 8.00 & 5.00 \\
0.9 & 38.28 & 18.00 & 10.00 \\
\hline
\end{tabular}

the scenarios described in Table 1 for $T$ and $X$, and for 3 levels of the Kendall's coefficient $\tau \in\{0.2,0.5,0.8\}$ (corresponding to the dependence parameter $\theta$ in Table 2). For comparison purpose, the in-control value of ATS is the same as in Rahali et al. ${ }^{29}$, i.e. ATS $_{0}=370$. For instance, in Table 3 , if $T$ follows a Gamma distribution with $\sigma_{0}=5$ and $X$ follows a Normal distribution with $\sigma_{0}=2$ (remember that $\mu_{0}=10$ for all the scenarios in Table 1 ) then $\mathrm{UCL}_{Z_{1}}=0.748$, 0.592 and 0.438 when $\tau=0.2,0.5$ and 0.8 , respectively. From Tables $3-5$, we can draw the following conclusions:

- When the statistic $Z \in\left\{Z_{1}, Z_{2}, Z_{3}\right\}$, the parameters in Table 1 and the type of Copula are fixed, the larger $\tau$ (i.e. the dependence between $T$ and $X$ ), the smaller the control limit $\mathrm{UCL}_{Z}$. This remark remains valid when a comparison is performed with Table 2 in Rahali et al. ${ }^{29}$ where the random variables $T$ and $X$ were assumed independent (which corresponds to the case $\tau=0$ ).

- With the parameters in Table 1 , the value of $\tau$ and the type of Copula are fixed, the upper control limits of the statistic $Z \in\left\{Z_{1}, Z_{2}, Z_{3}\right\}$ always satisfy $\mathrm{UCL}_{Z_{1}}<\mathrm{UCL}_{Z_{2}}<\mathrm{UCL}_{Z_{3}}$.

- With the parameters in Table 1 , the value of $\tau$ and statistic $Z \in\left\{Z_{1}, Z_{2}, Z_{3}\right\}$ are fixed, the upper control limits are more or less the same no matter the type of Copula considered.

Since the upper control limit in (15) depends on the choice of the distribution for $T$ and $X$ and also on the values for $\mu_{T_{0}}$ and $\mu_{X_{0}}$, it is important to evaluate the impact of the number $m$ of Phase I data used for estimating them and to provide some guidelines concerning the minimum acceptable value for $m$. Table 6 gives the $95 \%$ confidence intervals [ $\mathrm{UCL}_{Z, m}^{\mathrm{inf}}, \mathrm{UCL}_{Z, m}^{\mathrm{sup}}$ ] for $\mathrm{UCL}_{Z}$ (obtained using Monte Carlo simulations) and their relative differences

$$
\Delta=\frac{\mathrm{UCL}_{Z, m}^{\mathrm{sup}}-\mathrm{UCL}_{Z, m}^{\mathrm{inf}}}{\mathrm{UCL}_{Z, \infty}}
$$


as a function of $m \in\{20,50,100,200,500,1000,2000, \infty\}$, for the statistic $Z \in$ $\left\{Z_{1}, Z_{2}, Z_{3}\right\}$, for some combinations of the Gamma, Normal and Weibull distributions and for the dependence parameter $\tau \in\{0.2,0.8\}$ (Frank's Copula only). The smaller the value of $\Delta$, the more "accurate" is the estimation of the upper control limit $\mathrm{UCL}_{Z}$. The values of $\mathrm{UCL}_{Z, \infty}$ have already been computed in Table 3. As for the previous tables, we assume that $\mu_{T_{0}}=\mu_{X_{0}}=10$. The following conclusions can be drawn from Table 6 :

- when $m$ is small, the relative difference $\Delta$ can be very large. For instance, for the statistic $Z_{2}$, for the case of Weibull $\left(\sigma_{T_{0}}=5\right)$ and Normal $\left(\sigma_{X_{0}}=\right.$ 1 ), we have $\Delta=4.138$ when $m=20$ and $\tau=0.8$. As expected, when $m$ becomes large, the relative difference $\Delta$ converge to 0 (i.e. $\mathrm{UCL}_{Z, m}^{\mathrm{inf}}$ and $\mathrm{UCL}_{Z, m}^{\mathrm{sup}}$ both converge to $\left.\mathrm{UCL}_{Z, \infty}\right)$.

- The smaller the value of $\sigma_{T_{0}}$ for $T$, the better. Taking the same example (i.e. statistic $Z_{2}, m=20$ and $\tau=0.8$ ) but for the case of Gamma $\left(\sigma_{T_{0}}=1\right)$ and Normal $\left(\sigma_{X_{0}}=2\right)$, we have $\Delta=0.251$ (to be compared with $\Delta=4.138)$.

- When $\sigma_{T_{0}}$ is small, a better compromise is to choose the statistic $Z_{3}$ which yields smaller values for $\Delta$, while when $\sigma_{T_{0}}$ is large a better compromise is to choose the statistic $Z_{1}$.

- The value of the dependence parameter $\tau$ seems to have a medium impact on $\Delta$ (the difference between the cases $\tau=0.2$ and $\tau=0.8$ is not that large). In most cases, if we want to estimate the upper control limit $\mathrm{UCL}_{Z}$ accurately (say $\Delta \leq 0.05$ ), it seems that $m$ should be very large and, at least larger than 1000 .

When an upward shift occurs for $Z$, three possible situations are likely to occur:

- an upward shift in the amplitude $X$ from $\mu_{X_{0}}$ to $\mu_{X_{1}}=\delta_{X} \mu_{X_{0}}$ where $\delta_{X} \geq 1$ is the parameter quantifying the change in the amplitude,

- a downward shift in the time $T$ from $\mu_{T_{0}}$ to $\mu_{T_{1}}=\delta_{T} \mu_{T_{0}}$ where $\delta_{T} \leq 1$ is the parameter quantifying the change in the time,

- or also a change in both the amplitude $X$ from $\mu_{X_{0}}$ to $\mu_{X_{1}}=\delta_{X} \mu_{X_{0}}$ and in the time $T$ from $\mu_{T_{0}}$ to $\mu_{T_{1}}=\delta_{T} \mu_{T_{0}}$.

As in Rahali et al. ${ }^{29}$, we suggest to evaluate the performance of the proposed TBEA charts using

- the Expected Average Time to Signal, $\operatorname{EATS}_{X}$ for $X$ (assuming $\left.\delta_{T}=1\right)$ defined as

$$
\operatorname{EATS}_{X}=\sum_{\delta_{X} \in \Omega_{X}} f_{\delta_{X}}\left(\delta_{X}\right) \operatorname{ATS}\left(\delta_{X}, 1\right),
$$

- the Expected Average Time to Signal, $\operatorname{EATS}_{T}$ for $T$ (assuming $\delta_{X}=1$ ) defined as

$$
\operatorname{EATS}_{T}=\sum_{\delta_{T} \in \Omega_{T}} f_{\delta_{T}}\left(\delta_{T}\right) \operatorname{ATS}\left(1, \delta_{T}\right),
$$



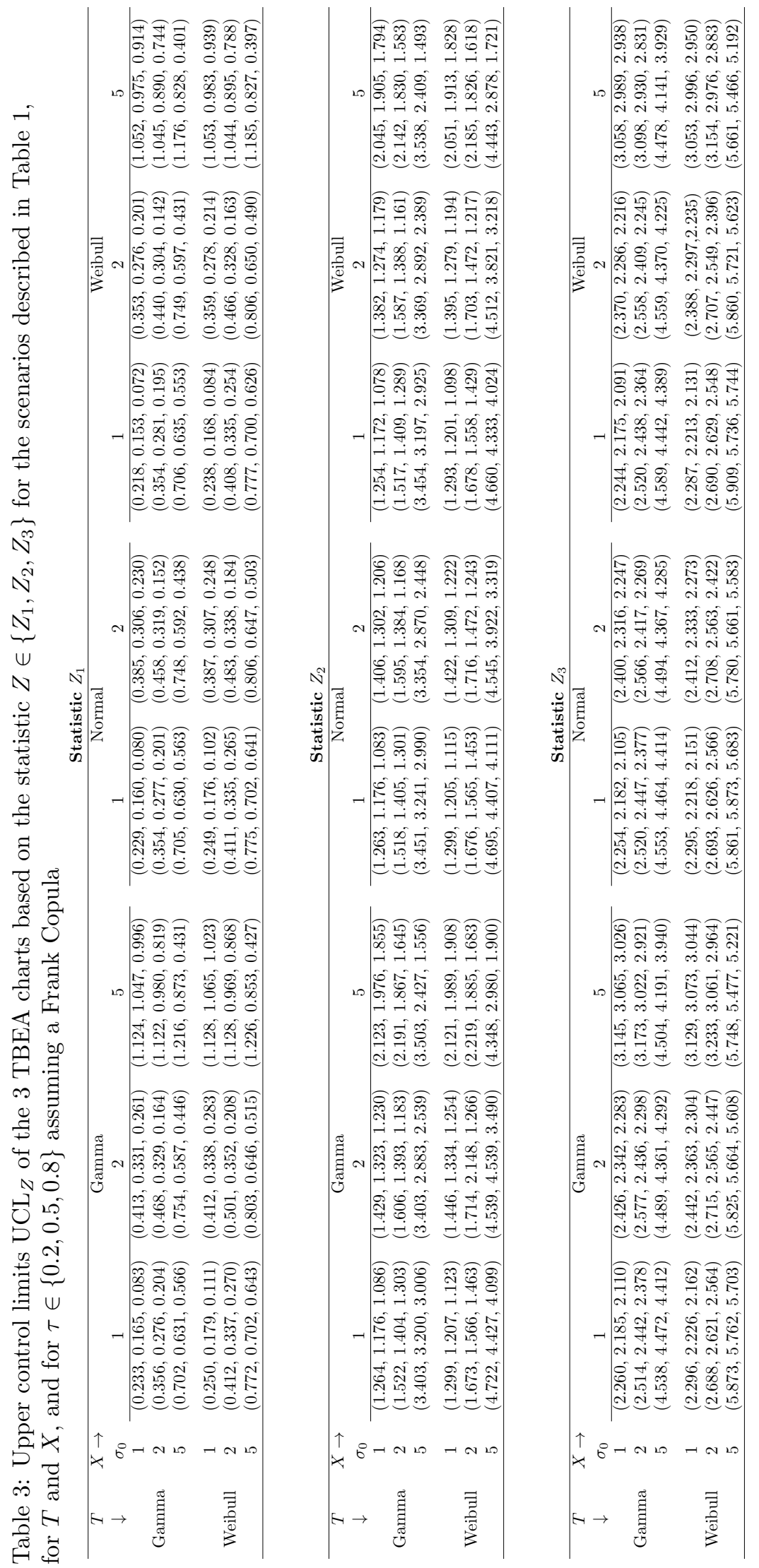

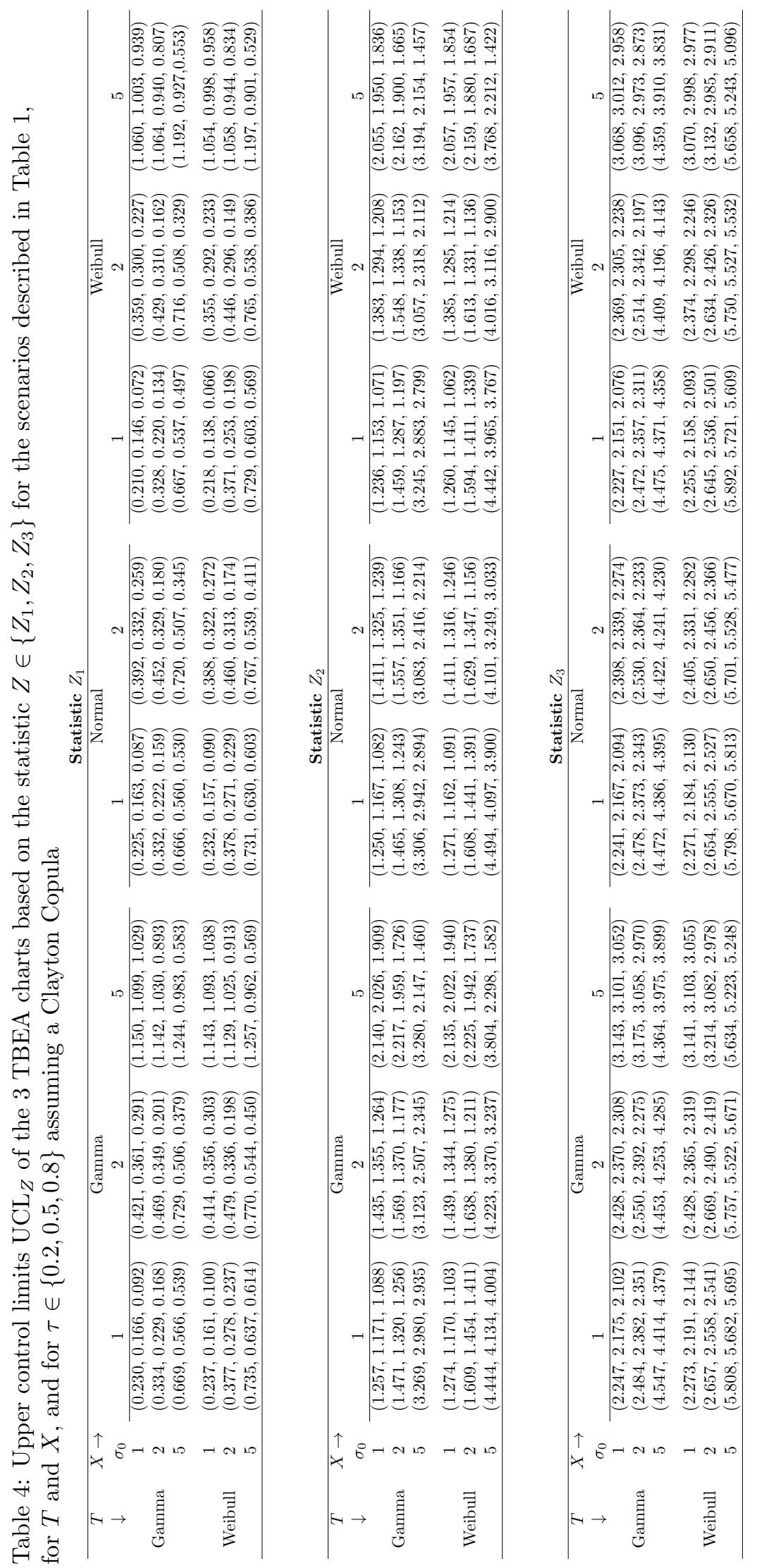

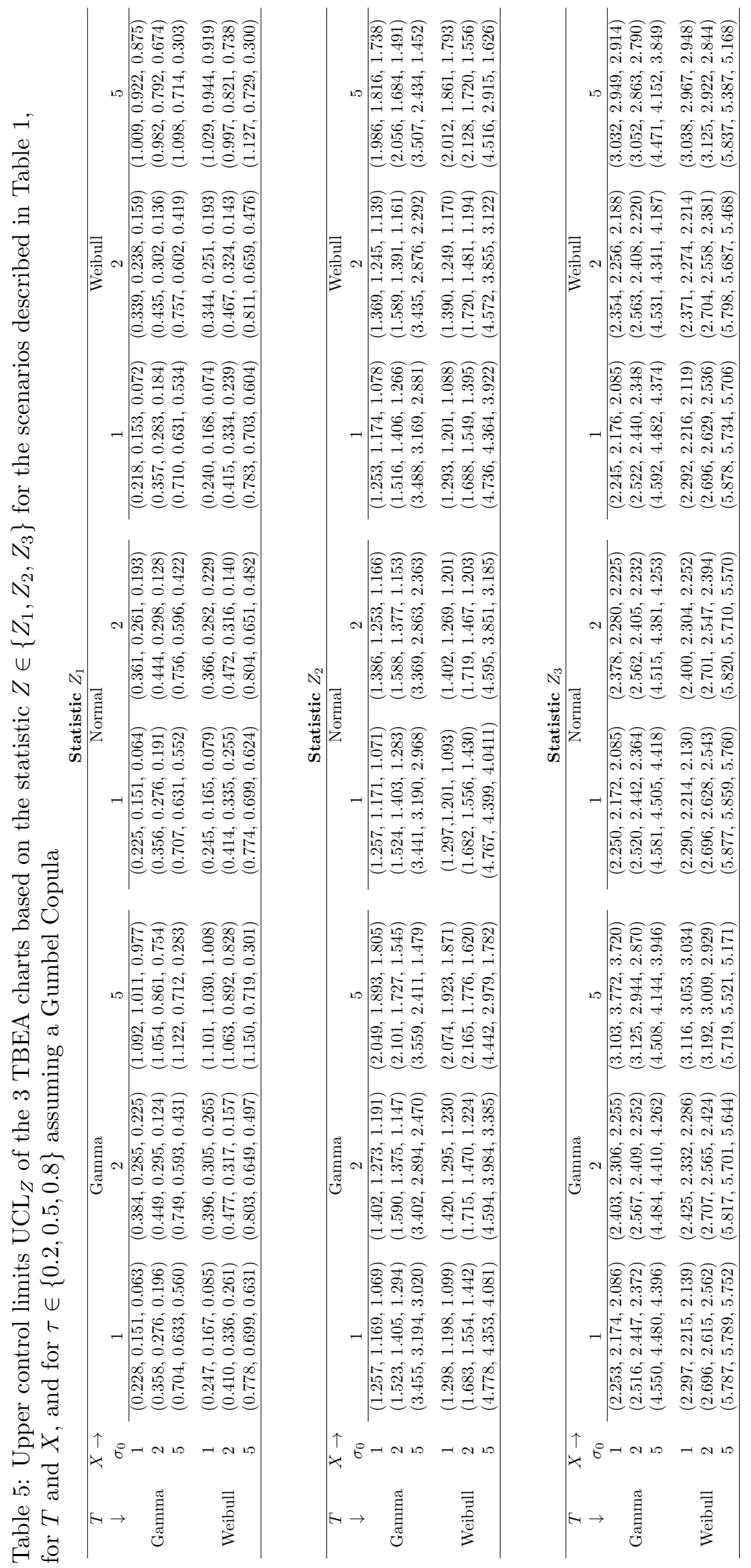
Table 6: $95 \%$ confidence intervals for $\mathrm{UCL}_{Z}$ and their relative differences $\Delta$ as a function of $m$, for the statistic $Z \in\left\{Z_{1}, Z_{2}, Z_{3}\right\}$, for some combinations of the Gamma, Normal and Weibull distributions and for the dependence parameter $\tau \in\{0.2,0.8\}$ (Frank's Copula only)

\begin{tabular}{|c|c|c|c|c|}
\hline & \multicolumn{4}{|c|}{ Statistic $Z_{1}$} \\
\hline$T \rightarrow$ & \multirow{2}{*}{\multicolumn{2}{|c|}{$\begin{array}{l}\text { Gamma }\left(\sigma_{T_{0}}=1\right) \\
\text { Normal }\left(\sigma_{X_{0}}=2\right)\end{array}$}} & \multicolumn{2}{|c|}{ Weibull $\left(\sigma_{T_{0}}=5\right)$} \\
\hline$X \rightarrow$ & & & Normal & $\left.X_{0}=1\right)$ \\
\hline$m$ & $\tau=0.2$ & $\tau=0.8$ & $\tau=0.2$ & $\tau=0.8$ \\
\hline 20 & {$[0.170,0.606], 1.132$} & {$[0.102,0.438], 1.460$} & {$[0.468,1.009], 0.698$} & {$[0.396,0.818], 0.658$} \\
\hline 50 & {$[0.280,0.580], 0.779$} & {$[0.149,0.417], 1.165$} & {$[0.622,0.993], 0.478$} & {$[0.521,0.784], 0.410$} \\
\hline 100 & {$[0.300,0.527], 0.589$} & {$[0.173,0.351], 0.773$} & {$[0.658,0.908], 0.322$} & {$[0.551,0.752], 0.313$} \\
\hline 200 & {$[0.320,0.470], 0.389$} & {$[0.180,0.306], 0.547$} & {$[0.695,0.866], 0.220$} & {$[0.566,0.719], 0.238$} \\
\hline 500 & {$[0.337,0.434], 0.251$} & {$[0.196,0.275], 0.343$} & {$[0.718,0.836], 0.152$} & {$[0.595,0.685], 0.140$} \\
\hline 1000 & {$[0.350,0.418], 0.176$} & {$[0.206,0.261], 0.239$} & {$[0.735,0.814], 0.101$} & {$[0.606,0.671], 0.101$} \\
\hline 2000 & {$[0.357,0.409], 0.135$} & {$[0.212,0.252], 0.173$} & {$[0.743,0.803], 0.077$} & {$[0.614,0.664], 0.078$} \\
\hline$\infty$ & {$[0.385,0.385], 0.000$} & {$[0.230,0.230], 0.000$} & {$[0.775,0.775], 0.000$} & {$[0.641,0.641], 0.000$} \\
\hline
\end{tabular}

Statistic $Z_{2}$

\begin{tabular}{|c|c|c|c|c|}
\hline & \multicolumn{4}{|c|}{ Statistic $Z_{2}$} \\
\hline$T \rightarrow$ & \multicolumn{2}{|c|}{$\operatorname{Gamma}\left(\sigma_{T_{0}}=1\right)$} & \multicolumn{2}{|c|}{ Weibull $\left(\sigma_{T_{0}}=5\right)$} \\
\hline$X \rightarrow$ & \multicolumn{2}{|c|}{ Normal $\left(\sigma_{X_{0}}=2\right)$} & \multicolumn{2}{|c|}{ Normal $\left(\sigma_{X_{0}}=1\right)$} \\
\hline$m$ & $\tau=0.2$ & $\tau=0.8$ & $\tau=0.2$ & $\tau=0.8$ \\
\hline 20 & {$[1.170,1.714], 0.386$} & {$[1.084,1.387], 0.251$} & {$[2.067,21.097], 4.053$} & {$[1.792,18.804], 4.138$} \\
\hline 50 & {$[1.287,1.694], 0.289$} & {$[1.134,1.379], 0.203$} & {$[2.748,20.836], 3.852$} & {$[2.551,18.738], 3.937$} \\
\hline 100 & {$[1.313,1.573], 0.184$} & {$[1.153,1.305], 0.126$} & {$[3.094,10.941], 1.671$} & {$[2.731,8.690], 1.449$} \\
\hline 200 & {$[1.332,1.513], 0.128$} & {$[1.164,1.267], 0.085$} & {$[3.494,7.435], 0.839$} & {$[3.008,6.659], 0.888$} \\
\hline 500 & {$[1.358,1.468], 0.078$} & {$[1.173,1.243], 0.058$} & {$[3.757,6.166], 0.513$} & {$[3.326,5.504], 0.529$} \\
\hline 1000 & {$[1.370,1.452], 0.058$} & {$[1.183,1.231], 0.039$} & {$[4.017,5.737], 0.366$} & {$[3.526,5.008], 0.360$} \\
\hline 2000 & {$[1.380,1.438], 0.041$} & {$[1.188,1.222], 0.028$} & {$[4.196,5.372], 0.250$} & {$[3.638,4.637], 0.243$} \\
\hline$\infty$ & {$[1.406,1.406], 0.000$} & {$[1.206,1.206], 0.000$} & {$[4.695,4.695], 0.000$} & {$[4.111,4.111], 0.000$} \\
\hline
\end{tabular}

Statistic $Z_{3}$

\begin{tabular}{|c|c|c|c|c|}
\hline & \\
\hline$T \rightarrow$ & \multirow{2}{*}{\multicolumn{2}{|c|}{$\begin{array}{l}\text { Gamma }\left(\sigma_{T_{0}}=1\right) \\
\text { Normal }\left(\sigma_{X_{0}}=2\right)\end{array}$}} & \multirow{2}{*}{\multicolumn{2}{|c|}{$\begin{array}{l}\text { Weibull }\left(\sigma_{T_{0}}=5\right) \\
\text { Normal }\left(\sigma_{X_{0}}=1\right)\end{array}$}} \\
\hline$X \rightarrow$ & & & & \\
\hline$m$ & $\tau=0.2$ & $\tau=0.8$ & $\tau=0.2$ & $\tau=0.8$ \\
\hline 20 & {$[2.199,2.648], 0.187$} & {$[2.110,2.453], 0.152$} & {$[2.927,24.828], 3.736$} & {$[2.936,24.298], 3.758$} \\
\hline 50 & {$[2.284,2.605], 0.133$} & {$[2.168,2.444], 0.122$} & {$[4.027,23.506], 3.323$} & {$[3.697,24.143], 3.597$} \\
\hline 100 & {$[2.312,2.538], 0.094$} & {$[2.184,2.360], 0.078$} & {$[4.162,12.156], 1.363$} & {$[4.118,12.259], 1.432$} \\
\hline 200 & {$[2.331,2.483], 0.063$} & {$[2.198,2.327], 0.057$} & {$[4.542,9.032], 0.766$} & {$[4.362,8.795], 0.780$} \\
\hline 500 & {$[2.354,2.450], 0.040$} & {$[2.214,2.293], 0.035$} & {$[4.876,7.392], 0.429$} & {$[4.821,7.415], 0.456$} \\
\hline 1000 & {$[2.362,2.434], 0.030$} & {$[2.224,2.280], 0.024$} & {$[5.070,6.912], 0.314$} & {$[4.969,6.871], 0.334$} \\
\hline 2000 & {$[2.374,2.424], 0.020$} & {$[2.230,2.271], 0.018$} & {$[5.289,6.564], 0.217$} & {$[5.223,6.400], 0.207$} \\
\hline$\infty$ & {$[2.400,2.400], 0.000$} & {$[2.247,2.247], 0.000$} & {$[5.861,5.861], 0.000$} & {$[5.683,5.683], 0.000$} \\
\hline
\end{tabular}


- the Expected Average Time to Signal, EATS for both $X$ and $T$ defined as

$$
\operatorname{EATS}_{X T}=\sum_{\delta_{X} \in \Omega_{X}} \sum_{\delta_{T} \in \Omega_{T}} f_{\delta_{X}}\left(\delta_{X}\right) f_{\delta_{T}}\left(\delta_{T}\right) A T S\left(\delta_{X}, \delta_{T}\right)
$$

where $\Omega_{X}$ and $\Omega_{T}$ are the "range of possible shifts" for $\delta_{X}$ and $\delta_{T}$, respectively, and $f_{\delta_{X}}\left(\delta_{X}\right)$ and $f_{\delta_{T}}\left(\delta_{T}\right)$ are the p.m.f. (probability mass functions) of the shifts $\delta_{X}$ and $\delta_{T}$ over $\Omega_{X}$ and $\Omega_{T}$, respectively. To study the effeciency of the uppersided TBEA control charts for an increase in the amplitude $X$ and / or a decrease in the time between events $T$, we will use the same situation discussed in Rahali et al. ${ }^{29}$ with $\Omega_{X}=\{1.1,1.2, \ldots, 1.9,2\}$ and $\Omega_{T}=\{0.5,0.55, \ldots, 0.9,0.95\}$.

As the results obtained in Tables 3-5 are quite similar, from now on, we will only present the resuts for the Frank Copulas. Results for the Clayton and the Gumbel Copulas have also been obtained (and can be requested from the corresponding author) but due to a lack of space, they will not be presented here. Results in Rahali et al. ${ }^{29}$ showed that, for independent $T$ and $X$, the choice of the statistic $\left(Z_{1}, Z_{2}\right.$ or $\left.Z_{3}\right)$ to be monitored clearly depends on the kind of expected shift:

- If the shift is due to a change in the amplitude $X$, the statistic $Z_{1}$ is the better choice as it allows us to obtain smaller $\operatorname{EATS}_{X}$ values.

- If the shift is due to a change in the time between events $T$, the statistic $Z_{3}$ is more appropriate as it allows us to obtain smaller $\operatorname{EATS}_{T}$ values.

- If the shift is due to a change in the amplitude $X$ and in the time between events $T$, the overall best option is the statistic $Z_{1}$ as it allows us to obtain smaller EATS $_{X T}$ values.

Now, we would like to investigate if these findings remain valid when a dependence exists between the time between events $T$ and the corresponding amplitude $X$ using the Frank Copula. For the same scenarios as in Table 1 and for the control limits in Table 3, the EATS $_{X T}$ values corresponding to $\tau \in\{0.2,0.5,0.8\}$ (i.e. small, medium and strong dependence) have been computed and listed in Tables 7, 8 and 9, respectively (results for $\mathrm{EATS}_{X}$ and $\mathrm{EATS}_{T}$ are not presented here but can also be requested from the corresponding author). From these tables, some interesting findings can be drawn:

- When the statistic $Z \in\left\{Z_{1}, Z_{2}, Z_{3}\right\}$ and the parameters in Table 1 are fixed, the larger the $\tau$, the smaller the values of $\operatorname{EATS}_{X T}$.

- For $\tau=0.2$ (see bold values in Table 7 ) the statistic $Z_{1}$ seems to be the best option as it gives the smallest EATS $_{X T}$ values (in $65 \%$ of the cases with an average $\mathrm{EATS}_{X T}$ value $\left.\overline{\mathrm{EATS}}_{X T}=14.46\right)$, followed by the statistic $Z_{2}$ (in $35 \%$ of the cases with an average $\operatorname{EATS}_{X T}$ value $\overline{\operatorname{EATS}}_{X T}=22.08$ ).

- For $\tau=0.5$ (see bold values in Table 8), the statistic $Z_{1}$ produces the smallest EATS $_{X T}$ values(in $67 \%$ of the cases with an average EATS $_{X T}$ value $\overline{\operatorname{EATS}}_{X T}=12.35$ ), followed by the statistic $Z_{2}$ (in $33 \%$ of the cases with an average $\operatorname{EATS}_{X T}$ value $\left.\overline{\operatorname{EATS}}_{X T}=16.34\right)$. 
- For $\tau=0.8$ (see bold values in Table 9), the most efficient statistic is $Z_{1}$ (in $67 \%$ of the cases with an average $\operatorname{EATS}_{X T}$ value $\overline{\operatorname{EATS}}_{X T}=9.74$ ), followed by $Z_{2}$ (in $33 \%$ of the cases with an average EATS $_{X T}$ value $\left.\overline{\operatorname{EATS}}_{X T}=13.16\right)$.

These results clearly show that, irrespective of the level of dependence, for a simultaneous change in the amplitude $X$ and in the time between events $T$, the overall best option is the statistic $Z_{1}$ or, eventually, the statistic $Z_{2}$ but the statistic $Z_{3}$ cannot be considered as potential efficient monitoring statistic. Whereas, in Rahali et al. ${ }^{29}$, the most efficient statistic is $Z_{1}$ with an average EATS value of $\overline{\mathrm{EATS}}=14.91$, followed by $Z_{2}$ with an average EATS value of $\overline{\mathrm{EATS}}=29.15$, and $Z_{3}$ with an average EATS value of $\overline{\mathrm{EATS}}=11.74$. This also allows us to conclude that the statistic $Z_{1}$ is the best option whether there is a dependence between $T$ and $X$ or not.

In order to clarify how much the dependence between $T$ and $X$ has a negative impact on the efficiency of the TBEA charts, we have also recomputed the $\overline{\operatorname{EATS}}_{X T}$ in the case of a Frank Copula with $\tau=0.5$ using the control limits obtained in Rahali et al. ${ }^{29}$ (instead of the ones provided in this paper), i.e. by assuming that $T$ and $X$ are actually independent random variables. The results are presented in Table 10 and, with a simple comparison with the results obtained in Table 8 , it can be seen that neglecting the dependence between $T$ and $X$ clearly increases the $\overline{\operatorname{EATS}}_{X T}$ values (by about $60 \%$ for $Z_{1}$ and by about $200 \%$ for $Z_{2}$, on the average). Similar results have also been obtained for $\tau=0.2$ and $\tau=0.8$ (these results are not presented in this paper but they can be requested from the corresponding author). As expected, the larger $\tau$ is, the stronger the negative impact on the TBEA charts. This clearly emphasizes the fact that using specific control limits by taking into account of the dependence between $T$ and $X$, improves the efficiency of the TBEA charts.

\section{Illustrative example}

For one of its bottleneck machine, a company recorded (see Table 11) from 08/01/12 to $27 / 12 / 18$ (format DD/MM/YY) all the breakdown dates (in days) as well as the estimated corresponding incurred costs $\left(X_{i}\right.$, in euros) which include all the repair and restart costs (spare parts, manpower) and the cost of manufacturing disruption. From these dates, it is easy to obtain the times between two consecutive breakdowns ( $T_{i}$ in days). For instance, the machine started to operate on $08 / 01 / 12$ for the first time, its first breakdown happened on $10 / 03 / 12$, i.e. $T_{1}=62$ days later, and the costs incurred for reparing and restarting the machine have been estimated as $X_{1}=4890$ euros. The data in Table 11 are divided into

- 30 breakdowns recorded for 5 years, from 2012 to 2016, and used here as a Phase I data set.

- 14 breakdowns recorded for 2 years, from 2017 to 2018, and used here as a Phase II data set.

In the reliability terminology, where the life of a machine is modeled as a "bathtub" curve, our Phase I must be considered as the constant failure rate period 
Table 7: EATS $_{X T}$ values for the 3 TBEA charts based on statistics $Z \in$ $\left\{Z_{1}, Z_{2}, Z_{3}\right\}$, Frank Copula, $\tau=0.2$

\begin{tabular}{|c|c|c|c|c|c|c|c|c|c|}
\hline \multirow{3}{*}{$\begin{array}{l}T \\
\downarrow\end{array}$} & \multirow{3}{*}{$\begin{array}{c}X \rightarrow \\
\sigma_{0}\end{array}$} & \multicolumn{8}{|c|}{ statistic $Z_{1}$} \\
\hline & & \multicolumn{3}{|c|}{ Gamma } & \multicolumn{2}{|c|}{ Normal } & \multicolumn{3}{|c|}{ Weibull } \\
\hline & & 1 & 2 & 5 & 1 & 2 & 1 & 2 & 5 \\
\hline \multirow{3}{*}{ Gamma } & 1 & 7.9 & 11.3 & 44.3 & 8.0 & 10.0 & 7.8 & 9.6 & 38.8 \\
\hline & 2 & 9.6 & 12.8 & 48.8 & 9.7 & 12.2 & 9.4 & 11.5 & 37.3 \\
\hline & 5 & 16.1 & 17.3 & 49.2 & 15.89 & 18.4 & 16.1 & 17.9 & 43.3 \\
\hline \multirow{3}{*}{ Weibull } & 1 & 8.2 & 11.3 & 50.8 & 8.1 & 10.6 & 8.2 & 9.8 & 36.5 \\
\hline & 2 & 11.4 & 13.3 & 47.3 & 11.3 & 12.8 & 11.4 & 12.4 & 35.7 \\
\hline & 5 & 18.3 & 21.4 & 43.4 & 17.8 & 21.0 & 18.5 & 20.1 & 42.3 \\
\hline
\end{tabular}

Statistic $Z_{2}$

\begin{tabular}{|c|c|c|c|c|c|c|c|c|c|}
\hline \multirow{3}{*}{$\begin{array}{c}T \\
\downarrow\end{array}$} & \multirow{3}{*}{$\begin{array}{c}X \rightarrow \\
\sigma_{0}\end{array}$} & \multicolumn{8}{|c|}{ Statist1c $Z_{2}$} \\
\hline & & \multicolumn{3}{|c|}{ Gamma } & \multicolumn{2}{|c|}{ Normal } & \multicolumn{3}{|c|}{ Weibull } \\
\hline & & 1 & 2 & 5 & 1 & 2 & 1 & 2 & 5 \\
\hline \multirow{3}{*}{ Gamma } & 1 & 8.1 & 10.1 & 8.0 & 8.1 & 9.6 & 7.9 & 9.2 & 25.4 \\
\hline & 2 & 11.2 & 13.2 & 27.3 & 11.1 & 12.5 & 11.1 & 12.4 & 26.2 \\
\hline & 5 & 32.5 & 31.2 & 38.8 & 30.7 & 34.1 & 32.6 & 32.1 & 38.0 \\
\hline \multirow{3}{*}{ Weibull } & 1 & 8.6 & 10.3 & 28.4 & 8.5 & 9.9 & 8.5 & 9.5 & 24.2 \\
\hline & 2 & 15.2 & 15.5 & 31.6 & 16.3 & 15.6 & 16.7 & 15.6 & 26.9 \\
\hline & 5 & 43.8 & 44.6 & 48.8 & 45.8 & 45.9 & 45.6 & 46.3 & 41.9 \\
\hline
\end{tabular}

Statistic $Z_{3}$

\begin{tabular}{|c|c|c|c|c|c|c|c|c|c|}
\hline$T$ & $X \rightarrow$ & \multicolumn{3}{|c|}{ Gamma } & \multicolumn{2}{|c|}{ Normal } & \multicolumn{3}{|c|}{ Weibull } \\
\hline \multirow[t]{2}{*}{$\downarrow$} & $\sigma_{0}$ & 1 & 2 & 5 & 1 & 2 & 1 & 2 & 5 \\
\hline & 1 & 8.0 & 10.6 & 40.5 & 8.0 & 9.7 & 7.9 & 11.1 & 29.6 \\
\hline \multirow[t]{3}{*}{ Gamma } & 2 & 11.5 & 13.5 & 37.0 & 11.3 & 12.5 & 12.0 & 12.2 & 26.5 \\
\hline & 5 & 50.2 & 47.9 & 49.9 & 55.9 & 49.3) & 52.6 & 49.3 & 51.3 \\
\hline & 1 & 8.7 & 10.7 & 38.8 & 8.73 & 10.2 & 8.5 & 9.6 & 32.7 \\
\hline \multirow[t]{2}{*}{ Weibull } & 2 & 18.2 & 17.4 & 37.7 & 17.7 & 17.6 & 17.9 & 16.8 & 29.3 \\
\hline & 5 & 73.4 & 84.9 & 72.4 & 78.3 & 72.4 & 75.3 & 68.8 & 72.3 \\
\hline
\end{tabular}


Table 8: EATS $_{X T}$ values for the 3 TBEA charts based on statistics $Z \in$ $\left\{Z_{1}, Z_{2}, Z_{3}\right\}$, Frank Copula, $\tau=0.5$

\begin{tabular}{|c|c|c|c|c|c|c|c|c|c|}
\hline \multirow{3}{*}{$\begin{array}{l}T \\
\downarrow\end{array}$} & \multirow{3}{*}{$\begin{array}{c}X \rightarrow \\
\sigma_{0}\end{array}$} & \multicolumn{8}{|c|}{ Statistic $Z_{1}$} \\
\hline & & \multicolumn{3}{|c|}{ Gamma } & \multicolumn{2}{|c|}{ Normal } & \multicolumn{3}{|c|}{ Weibull } \\
\hline & & 1 & 2 & 5 & 1 & 2 & 1 & 2 & 5 \\
\hline \multirow{3}{*}{ Gamma } & 1 & 7.4 & 9.8 & 43.0 & 7.4 & 9.1 & 7.4 & 8.5 & 37.4 \\
\hline & 2 & 8.4 & 9.4 & 43.9 & 8.5 & 9.2 & 8.5 & 9.0 & 29.8 \\
\hline & 5 & 14.1 & 13.4 & 31.9 & 14.1 & 13.9 & 14.1 & 13.8 & 27.6 \\
\hline \multirow{3}{*}{ Weibull } & 1 & 7.6 & 10.1 & 43.3 & 7.5 & 9.2 & 7.6 & 8.6 & 34.4 \\
\hline & 2 & 9.9 & 10.2 & 43.3 & 9.5 & 9.5 & 10.1 & 9.4 & 29.9 \\
\hline & 5 & 16.4 & 15.3 & 29.1 & 15.9 & 15.9 & 16.8 & 16.0 & 29.8 \\
\hline
\end{tabular}

Statistic $Z_{2}$

\begin{tabular}{|c|c|c|c|c|c|c|c|c|c|}
\hline \multirow{3}{*}{$\begin{array}{c}T \\
\downarrow\end{array}$} & \multirow{3}{*}{$\begin{array}{c}X \rightarrow \\
\sigma_{0}\end{array}$} & \multicolumn{8}{|c|}{ Statist1c $Z_{2}$} \\
\hline & & \multicolumn{3}{|c|}{ Gamma } & \multicolumn{2}{|c|}{ Normal } & \multicolumn{3}{|c|}{ Weibull } \\
\hline & & 1 & 2 & 5 & 1 & 2 & 1 & 2 & 5 \\
\hline \multirow{3}{*}{ Gamma } & 1 & 7.5 & 8.7 & 7.5 & 7.5 & 8.4 & 7.5 & 8.0 & 21.7 \\
\hline & 2 & 9.9 & 9.8 & 22.6 & 9.4 & 9.3 & 9.6 & 9.3 & 18.3 \\
\hline & 5 & 30.6 & 26.2 & 24.1 & 29.1 & 26.5 & 27.7 & 26.9 & 23.9 \\
\hline \multirow{3}{*}{ Weibull } & 1 & 7.7 & 8.9 & 26.9 & 7.7 & 8.4 & 7.6 & 8.2 & 20.4 \\
\hline & 2 & 13.7 & 11.4 & 24.2 & 12.5 & 11.3 & 13.2 & 12.3 & 21.2 \\
\hline & 5 & 43.5 & 40.9 & 32.4 & 42.5 & 39.4 & 40.6 & 41.5 & 32.1 \\
\hline
\end{tabular}

Statistic $Z_{3}$

\begin{tabular}{|c|c|c|c|c|c|c|c|c|c|}
\hline \multirow{3}{*}{$\begin{array}{l}T \\
\downarrow\end{array}$} & \multirow{3}{*}{$\begin{array}{c}X \rightarrow \\
\sigma_{0}\end{array}$} & \multicolumn{8}{|c|}{2} \\
\hline & & \multicolumn{3}{|c|}{ Gamma } & \multicolumn{2}{|c|}{ Normal } & \multicolumn{3}{|c|}{ Weibull } \\
\hline & & 1 & 2 & 5 & 1 & 2 & 1 & 2 & 5 \\
\hline \multirow{3}{*}{ Gamma } & 1 & 7.5 & 9.6 & 41.0 & 7.5 & 8.7 & 7.4 & 9.5 & 31.0 \\
\hline & 2 & 10.5 & 10.0 & 30.8 & 10.2 & 9.9 & 10.5 & 9.9 & 27.9 \\
\hline & 5 & 47.6 & 47.8 & 55.5 & 51.3 & 42.3 & 54.9 & 45.8 & 43.8 \\
\hline \multirow{3}{*}{ Weibull } & 1 & 7.9 & 9.8 & 37.9 & 7.7 & 8.9 & 7.7 & 8.3 & 34.6 \\
\hline & 2 & 15.9 & 15.0 & 32.2 & 16.3 & 13.9 & 16.8 & 14.7 & 26.2 \\
\hline & 5 & 72.8 & 71.9 & 67.9 & 70.9 & 74.0 & 67.1 & 77.3 & 71.7 \\
\hline
\end{tabular}


Table 9: EATS $_{X T}$ values for the 3 TBEA charts based on statistics $Z \in$ $\left\{Z_{1}, Z_{2}, Z_{3}\right\}$, Frank Copula, $\tau=0.8$

Statistic $Z_{1}$

\begin{tabular}{|c|c|c|c|c|c|c|c|c|c|}
\hline$T$ & $X \rightarrow$ & \multicolumn{3}{|c|}{ Gamma } & \multicolumn{2}{|c|}{ Normal } & \multicolumn{3}{|c|}{ Weibull } \\
\hline \multirow[t]{2}{*}{$\downarrow$} & $\sigma_{0}$ & 1 & 2 & 5 & 1 & 2 & 1 & 2 & 5 \\
\hline & 1 & 7.3 & 8.6 & 40.7 & 7.3 & 8.1 & 7.3 & 7.7 & 31.9 \\
\hline \multirow[t]{3}{*}{ Gamma } & 2 & 7.7 & 7.4 & 31.3 & 7.7 & 7.4 & 7.6 & 7.4 & 26.9 \\
\hline & 5 & 12.5 & 10.4 & 13.5 & 12.3 & 10.2 & 11.9 & 13.8 & 11.8 \\
\hline & 1 & 7.3 & 9.2 & 40.6 & 7.3 & 8.3 & 7.3 & 7.8 & 33.5 \\
\hline \multirow[t]{2}{*}{ Weibull } & 2 & 8.9 & 7.9 & 37.5 & 8.6 & 7.6 & 8.2 & 7.4 & 30.1 \\
\hline & 5 & 14.5 & 12.4 & 13.3 & 14.6 & 11.4 & 13.5 & 11.4 & 11.8 \\
\hline
\end{tabular}

Statistic $Z_{2}$

\begin{tabular}{|c|c|c|c|c|c|c|c|c|c|}
\hline \multirow{3}{*}{$\begin{array}{c}T \\
\downarrow\end{array}$} & \multirow{3}{*}{$\begin{array}{c}X \rightarrow \\
\sigma_{0}\end{array}$} & \multicolumn{8}{|c|}{ Nours } \\
\hline & & \multicolumn{3}{|c|}{ Gamma } & \multicolumn{2}{|c|}{ Normal } & \multicolumn{3}{|c|}{ Weibull } \\
\hline & & 1 & 2 & 5 & 1 & 2 & 1 & 2 & 5 \\
\hline \multirow{3}{*}{ Gamma } & 1 & 7.3 & 7.9 & 7.3 & 7.3 & 7.6 & 7.3 & 7.5 & 19.9 \\
\hline & 2 & 8.4 & 7.5 & 17.7 & 8.2 & 7.4 & 8.2 & 7.4 & 14.5 \\
\hline & 5 & 28.9 & 22.8 & 11.7 & 26.6 & 24.0 & 25.3 & 20.1 & 10.1 \\
\hline \multirow{3}{*}{ Weibull } & 1 & 7.3 & 8.3 & 24.5 & 7.2 & 7.8 & 7.3 & 7.5 & 21.3 \\
\hline & 2 & 11.3 & 8.4 & 20.6 & 10.7 & 7.9 & 10.2 & 7.8 & 16.3 \\
\hline & 5 & 39.0 & 34.8 & 18.0 & 42.9 & 32.8 & 37.1 & 32.9 & 14.8 \\
\hline
\end{tabular}

Statistic $Z_{3}$

\begin{tabular}{|c|c|c|c|c|c|c|c|c|c|}
\hline$T$ & $X \rightarrow$ & \multicolumn{3}{|c|}{ Gamma } & \multicolumn{2}{|c|}{ Normal } & \multicolumn{3}{|c|}{ Weibull } \\
\hline$\downarrow$ & $\sigma_{0}$ & 1 & 2 & 5 & 1 & 2 & 1 & 2 & 5 \\
\hline \multirow{3}{*}{ Gamma } & 1 & 7.3 & 8.4 & 41.9 & 7.3 & 8.0 & 7.3 & 9.1 & 32.6 \\
\hline & 2 & 9.7 & 8.6 & 30.5 & 9.7 & 8.0 & 9.5 & 7.8 & 24.5 \\
\hline & 5 & 56.6 & 48.0 & 49.9 & 53.9 & 46.7 & 45.0 & 47.8 & 48.7 \\
\hline \multirow{3}{*}{ Weibull } & 1 & 7.4 & 9.2 & 41.5 & 7.3 & 8.3 & 7.3 & 7.8 & 31.4 \\
\hline & 2 & 16.1 & 11.8 & 35.9 & 14.6 & 12.0 & 14.9 & 10.9 & 24.7 \\
\hline & 5 & 70.8 & 73.6 & 75.1 & 72.7 & 65.3 & 66.8 & 71.6 & 70.9 \\
\hline
\end{tabular}


Table 10: EATS $_{X T}$ values for the 3 TBEA charts based on statistics $Z \in$ $\left\{Z_{1}, Z_{2}, Z_{3}\right\}$ for a Frank Copula with $\tau=0.5$ using the control limits obtained in Rahali et al. ${ }^{29}$, i.e. by assuming that $T$ and $X$ are actually independent random variables

\begin{tabular}{|c|c|c|c|c|c|c|c|c|c|}
\hline \multirow{2}{*}{$T$} & \multirow{2}{*}{$X \rightarrow$} & \multicolumn{8}{|c|}{ Statistic $Z_{1}$} \\
\hline & & \multicolumn{3}{|c|}{ Gamma } & \multicolumn{2}{|c|}{ Normal } & \multicolumn{3}{|c|}{ Weibull } \\
\hline$\downarrow$ & $\sigma_{0}$ & 1 & 2 & 5 & 1 & 2 & 1 & 2 & 5 \\
\hline \multirow{3}{*}{ Gamma } & 1 & 9.5 & 16.4 & 62.3 & 9.4 & 14.7 & 8.7 & 13.4 & 51.0 \\
\hline & 2 & 13.8 & 29.6 & 81.7 & 13.4 & 29.3 & 12.6 & 26.5 & 74.0 \\
\hline & 5 & 22.4 & 60.7 & 188.2 & 22.7 & 54.8 & 22.4 & 52.2 & 197.3 \\
\hline \multirow{3}{*}{ Weibull } & 1 & 10.2 & 16.9 & 58.9 & 10.2 & 15.4 & 9.7 & 14.2 & 50.8 \\
\hline & 2 & 16.2 & 36.6 & 87.5 & 16.4 & 34.7 & 15.7 & 30.5 & 78.7 \\
\hline & 5 & 26.6 & 72.2 & 209.6 & 26.2 & 65.1 & 26.7 & 59.6 & 229.8 \\
\hline
\end{tabular}

\begin{tabular}{|c|c|c|c|c|c|c|c|c|c|}
\hline & & & & & Stati & ic $Z_{2}$ & & & \\
\hline$T$ & $X \rightarrow$ & & $\overline{\text { Gamn }}$ & & $\mathrm{No}$ & nal & & Weibu & \\
\hline$\downarrow$ & $\sigma_{0}$ & 1 & 2 & 5 & 1 & 2 & 1 & 2 & 5 \\
\hline & 1 & 9.8 & 16.3 & 51.1 & 9.4 & 15.4 & 8.9 & 14.3 & 44.1 \\
\hline Gamma & 2 & 14.5 & 28.9 & 98.9 & 14.4 & 27.2 & 14.4 & 24.8 & 99.3 \\
\hline & 5 & 37.9 & 45.9 & 108.8 & 37.7 & 47.6 & 38.3 & 46.3 & 110.1 \\
\hline & 1 & 10.9 & 18.0 & 49.7 & 10.8 & 17.1 & 10.4 & 16.4 & 45.9 \\
\hline Weibull & 2 & 20.1 & 33.1 & 108.5 & 21.2 & 32.3 & 20.7 & 31.1 & 124.7 \\
\hline & 5 & 53.4 & 61.6 & 108.5 & 52.9 & 61.9 & 54.4 & 64.1 & 112.5 \\
\hline
\end{tabular}

\begin{tabular}{|c|c|c|c|c|c|c|c|c|c|}
\hline & & \multicolumn{8}{|c|}{ Statistic $Z_{3}$} \\
\hline$T$ & $X \rightarrow$ & \multicolumn{3}{|c|}{ Gamma } & \multicolumn{2}{|c|}{ Normal } & \multicolumn{3}{|c|}{ Weibull } \\
\hline \multirow[t]{2}{*}{$\downarrow$} & $\sigma_{0}$ & 1 & 2 & 5 & 1 & 2 & 1 & 2 & 5 \\
\hline & 1 & 9.4 & 15.3 & 54.4 & 9.3 & 14.1 & 8.9 & 13.0 & 44.9 \\
\hline \multirow[t]{3}{*}{ Gamma } & 2 & 15.1 & 22.6 & 66.9 & 14.8 & 22.8 & 14.5 & 21.9 & 63.5 \\
\hline & 5 & 53.2 & 58.2 & 75.6 & 54.7 & 58.2 & 54.9 & 59.9 & 75.0 \\
\hline & 1 & 10.7 & 16.2 & 51.8 & 10.7 & 15.3 & 10.5 & 14.6 & 45.4 \\
\hline \multirow[t]{2}{*}{ Weibull } & 2 & 22.5 & 29.9 & 70.8 & 22.5 & 29.7 & 22.3 & 28.6 & 69.9 \\
\hline & 5 & 74.6 & 82.1 & 88.1 & 75.0 & 79.8 & 78.7 & 78.4 & 88.8 \\
\hline
\end{tabular}


of the machine, while our Phase II period will be considered as the beginning of its potentially increasing failure rate period (this will be confirmed later on). The first part of the "bathtub" curve, corresponding to a decreasing failure rate period is not presented here, i.e. all the early failures have been fixed and there is no report about this relatively short period.

The data, $T_{i}$ and $X_{i}$ in Table 11, have also been plotted in Figure 3 with $(\circ)$ and $(\bullet)$ corresponding to Phases I and II, respectively. From the bottommost plot of Figure 3, a slight positive correlation between the time $T$ between consecutive breakdowns and the corresponding incurred costs $X$ can be seen. More precisely, it seems that when the time between consecutive breakdowns $T$ is smaller (larger), the corresponding cost seems to be also smaller (larger). Investigations (during the period 2012 to 2016) about this phenomena have shown that, when a breakdown occurs and is fixed, then if the next breakdown occurs after a short period of time, it is often (but not always) due to similar causes and, consequently, i) the time for searching the breakdown causes are reduced and ii) the spare parts costs are also reduced as they have already been purchased for the previous breakdown. On the contrary, when the next breakdown occurs after a long period of time, the causes are usually different from the previous breakdown and need i) more time to be searched and ii) new spare parts to be purchased. In order to analytically confirm this fact, the Kendall's and Spearman's rank correlation coefficients have been computed, $\tau=0.4657$ and $\rho=0.6129$ along with their correponding $p$-values 0.00035 and 0.00032 , respectively, confirming a positive correlation between $T$ and $X$. In the case of a Frank Copula, the relationship between $\theta$ and $\tau$ is given by equations (27) and (28). As a consequence, if $\tau$ is known, then $\theta$ can be obtained by numerically solving the following equation:

$$
\tau-1-\frac{4}{\theta} \times\left(\frac{1}{\theta} \int_{0}^{\theta} \frac{t}{e^{t}-1} \mathrm{~d} t-1\right)=0 .
$$

In our case, for $\tau=0.4657$, we have $\theta=5.14$. In Table 12 , we provide the estimated values for $\mu_{T_{0}}, \sigma_{T_{0}}, \mu_{X_{0}}$ and $\sigma_{X_{0}}$, as well as the estimated values for $a_{0}$ and $b_{0}$ for the distributions considered in Table 1 . Based on the KolmogorovSmirnov distance $D_{K S}$ (the smaller, the better) the Gamma distribution will be chosen for the time $T$ and the Weibull distribution for the cost $X$.

Assuming an in-control ATS value, $\mathrm{ATS}_{0}=9125$ days (i.e. 25 years), the upper control limits of the 3 TBEA charts based on statistics $Z_{1}, Z_{2}$ and $Z_{3}$ are equal to $\mathrm{UCL}_{Z_{1}}=0.57, \mathrm{UCL}_{Z_{2}}=2.06$ and $\mathrm{UCL}_{Z_{3}}=3.18$, respectively. The TBEA charts, corresponding to the statistics $Z_{1}, Z_{2}$ and $Z_{3}$, are plotted in Figure 4 along with the upper control limits $\mathrm{UCL}_{Z_{1}}=0.57, \mathrm{UCL}_{Z_{2}}=2.06$ and $\mathrm{UCL}_{Z_{3}}=3.18$. As it can be seen, the Phase I part of these charts seems to confirm the fact that during the period from 2012 to 2016 , the time between consecutive breakdowns and their corresponding costs were a stable process. But, from 2017, things seems to have changed as several out-of-control situations (see values in bold in Table 11) have been detected by the 3 TBEA charts on $14 / 05 / 18,24 / 11 / 18$ and $27 / 12 / 18$ due to more frequent breakdowns and an increasing maintenance cost (due to an aging machine). Every time an outof-control situation is detected, the production is stopped, the sources of the 
Table 11: Phase I and II data sets corresponding to the time ( $T_{i}$ in days) between two consecutive breakdowns, amplitudes $\left(X_{i}\right.$ as the repair and restart cost in euros) and the values of the statistics $Z_{1}, Z_{2}$ and $Z_{3}$.

\begin{tabular}{|c|c|c|c|c|c|c|c|c|c|c|c|c|c|}
\hline \multicolumn{7}{|c|}{ Phase I } & \multicolumn{7}{|c|}{ Phase II } \\
\hline Date & $i$ & $T_{i}$ & $X_{i}$ & $Z_{1, i}$ & $Z_{2, i}$ & $Z_{3, i}$ & Date & $i$ & $T_{i}$ & $X_{i}$ & $Z_{1, i}$ & $Z_{2, i}$ & $Z_{3, i}$ \\
\hline $10 / 03 / 12$ & 1 & 62 & 4890 & -0.064 & 0.939 & 1.939 & $11 / 01 / 17$ & 1 & 63 & 5080 & -0.043 & 0.960 & 1.962 \\
\hline $28 / 05 / 12$ & 2 & 79 & 6180 & -0.092 & 0.932 & 1.995 & $21 / 03 / 17$ & 2 & 69 & 5350 & -0.090 & 0.923 & 1.935 \\
\hline $25 / 07 / 12$ & 3 & 58 & 3730 & -0.231 & 0.766 & 1.770 & $07 / 05 / 17$ & 3 & 47 & 3770 & -0.036 & 0.955 & 2.015 \\
\hline $27 / 08 / 12$ & 4 & 33 & 2930 & 0.032 & 1.057 & 2.377 & $15 / 07 / 17$ & 4 & 69 & 4590 & -0.243 & 0.792 & 1.782 \\
\hline $20 / 11 / 12$ & 5 & 85 & 7600 & 0.093 & 1.065 & 2.230 & $14 / 10 / 17$ & 5 & 91 & 5940 & -0.344 & 0.777 & 1.848 \\
\hline $20 / 02 / 13$ & 6 & 92 & 5580 & -0.434 & 0.722 & 1.768 & $18 / 12 / 17$ & 6 & 65 & 5420 & -0.008 & 0.993 & 2.002 \\
\hline $30 / 04 / 13$ & 7 & 69 & 4570 & -0.247 & 0.789 & 1.778 & $26 / 02 / 18$ & 7 & 70 & 4580 & -0.262 & 0.779 & 1.767 \\
\hline $06 / 07 / 13$ & 8 & 67 & 5230 & -0.080 & 0.930 & 1.937 & $21 / 04 / 18$ & 8 & 54 & 5430 & 0.181 & 1.197 & 2.189 \\
\hline $18 / 08 / 13$ & 9 & 43 & 4470 & 0.174 & 1.238 & 2.274 & $14 / 05 / 18$ & 9 & 23 & 5740 & 0.770 & 2.972 & 3.721 \\
\hline $28 / 09 / 13$ & 10 & 41 & 3420 & -0.005 & 0.993 & 2.128 & $27 / 06 / 18$ & 10 & 44 & 6110 & 0.488 & 1.654 & 2.574 \\
\hline $22 / 11 / 13$ & 11 & 55 & 3460 & -0.234 & 0.749 & 1.770 & $22 / 08 / 18$ & 11 & 56 & 7340 & 0.533 & 1.561 & 2.536 \\
\hline $08 / 02 / 14$ & 12 & 78 & 5360 & -0.241 & 0.818 & 1.839 & $29 / 10 / 18$ & 12 & 68 & 8160 & 0.495 & 1.429 & 2.516 \\
\hline $05 / 04 / 14$ & 13 & 56 & 4470 & -0.047 & 0.951 & 1.956 & $24 / 11 / 18$ & 13 & 26 & 4800 & 0.529 & 2.199 & 3.236 \\
\hline $28 / 05 / 14$ & 14 & 53 & 4470 & 0.004 & 1.004 & 2.015 & $27 / 12 / 18$ & 14 & 33 & 6570 & 0.768 & 2.371 & 3.113 \\
\hline $08 / 07 / 14$ & 15 & 41 & 3320 & -0.025 & 0.964 & 2.108 & & & & & & & \\
\hline $27 / 09 / 14$ & 16 & 81 & 4910 & -0.382 & 0.722 & 1.720 & & & & & & & \\
\hline $29 / 10 / 14$ & 17 & 32 & 5010 & 0.470 & 1.864 & 2.854 & & & & & & & \\
\hline $07 / 01 / 15$ & 18 & 70 & 6630 & 0.152 & 1.128 & 2.182 & & & & & & & \\
\hline $30 / 03 / 15$ & 19 & 82 & 5710 & -0.238 & 0.829 & 1.873 & & & & & & & \\
\hline $20 / 05 / 15$ & 20 & 51 & 5130 & 0.171 & 1.198 & 2.192 & & & & & & & \\
\hline $16 / 07 / 15$ & 21 & 57 & 5330 & 0.110 & 1.114 & 2.111 & & & & & & & \\
\hline $01 / 09 / 15$ & 22 & 47 & 5010 & 0.215 & 1.269 & 2.266 & & & & & & & \\
\hline $22 / 10 / 15$ & 23 & 51 & 3660 & -0.126 & 0.855 & 1.895 & & & & & & & \\
\hline $15 / 11 / 15$ & 24 & 24 & 3340 & 0.268 & 1.657 & 3.129 & & & & & & & \\
\hline $12 / 01 / 16$ & 25 & 58 & 3600 & -0.257 & 0.739 & 1.743 & & & & & & & \\
\hline $14 / 03 / 16$ & 26 & 62 & 5560 & 0.072 & 1.068 & 2.074 & & & & & & & \\
\hline $28 / 04 / 16$ & 27 & 45 & 5760 & 0.401 & 1.524 & 2.473 & & & & & & & \\
\hline $25 / 06 / 16$ & 28 & 58 & 6440 & 0.317 & 1.322 & 2.318 & & & & & & & \\
\hline $16 / 08 / 16$ & 29 & 52 & 6310 & 0.393 & 1.445 & 2.408 & & & & & & & \\
\hline 09/11/16 & 30 & 85 & 6300 & -0.169 & 0.883 & 1.967 & & & & & & & \\
\hline
\end{tabular}

Table 12: Estimated values for $\mu_{T_{0}}, \sigma_{T_{0}}, \mu_{X_{0}}$ and $\sigma_{X_{0}}$ as well as the estimated values for $a_{0}$ and $b_{0}$ for the distributions in Table 1.

\begin{tabular}{ccccccccc}
\cline { 2 - 4 } \cline { 6 - 8 } & \multicolumn{3}{c}{$T$} & & \multicolumn{3}{c}{$X$} \\
& $\mu_{T_{0}}=58.9, \sigma_{T_{0}}=17.28$ & & $\mu_{X_{0}}=4946, \sigma_{X_{0}}=1165.43$ \\
Gamma & $a_{0}$ & $b_{0}$ & $D_{K S}$ & & $a_{0}$ & $b_{0}$ & $D_{K S}$ \\
\cline { 2 - 4 } \cline { 6 - 8 } Normal & 58.9000 & 17.0562 & $\mathbf{0 . 0 8 1 8}$ & & 18.0107 & 274.6135 & 0.1228 \\
Weibull & 3.8123 & 65.1584 & 0.1264 & & 4.8472 & 5396.4958 & $\mathbf{0 . 1 1 2 9}$ \\
\hline
\end{tabular}



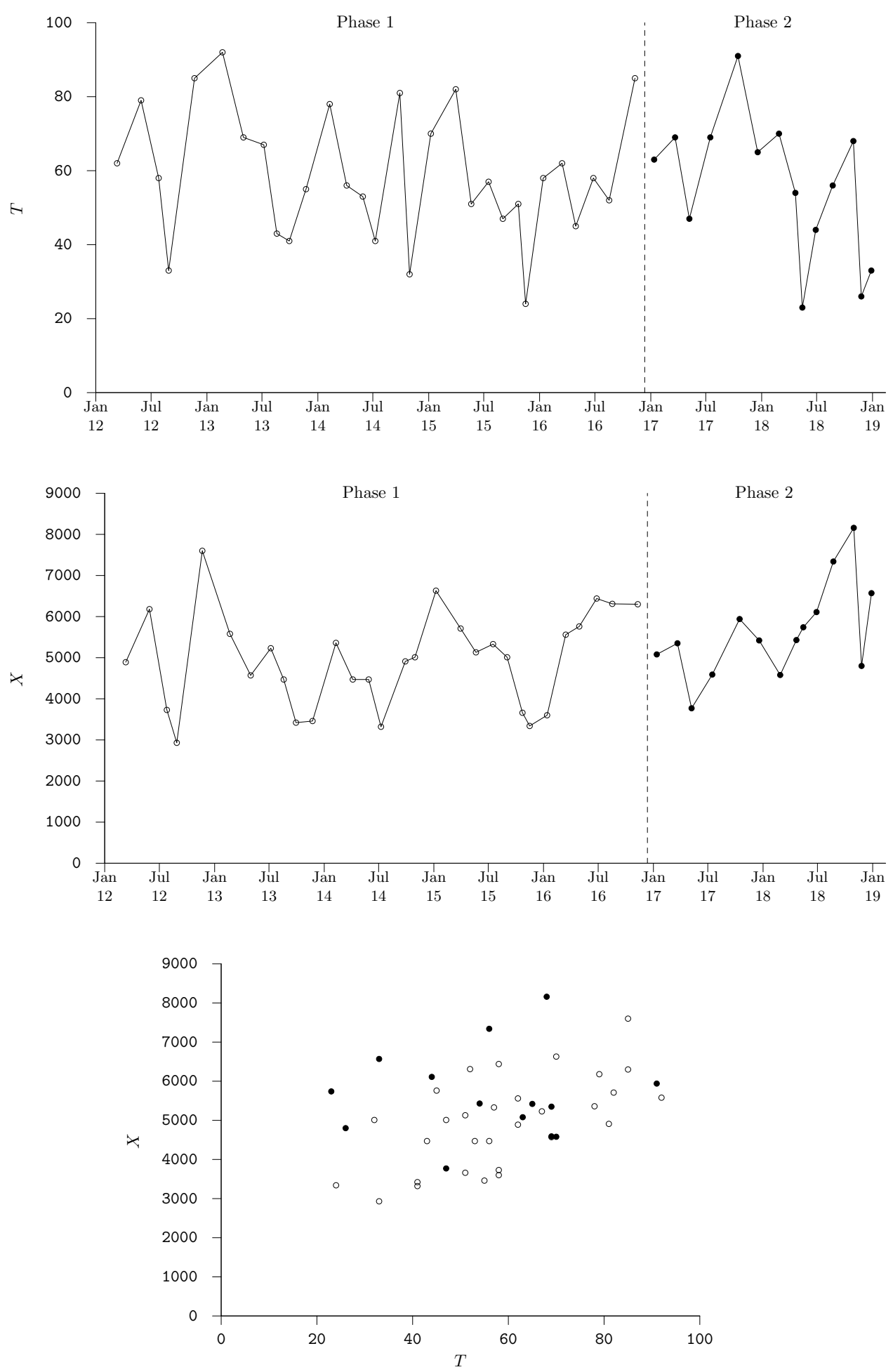

Figure 3: Phase I (o) and II $(\bullet)$ data corresponding to the time ( $T$ in days) between breakdowns and amplitudes ( $X$ as the repair and restart cost in euros) corresponding to the data set in Table 11. 
breakdown are searched for, analyzed and repaired. The machine is restarted as soon as possible in order to decrease the manufacturing disruption.

\section{Conclusions}

In this paper, we investigated simple Shewhart type control charts for several statistics and many distributional scenarios for simultaneously monitoring TBEA data. The Frank Copulas has been used as a mechanism to model the dependence between the time $T$ and the amplitude $X$. The main result of this paper is that, for a simultaneous change in the amplitude $X$ and in the time between events $T$, the overall best choice is the statistic $Z_{1}$ followed by $Z_{2}$, regardless of the level of dependence. On the contrary, the statistic $Z_{3}$ should not be considered as a potentially efficient statistic for monitoring.

In terms of potential future research, TBEA type control charts could be extended, for instance, in order to be able to monitor resilience type data which are known to depend on three characteristics: the time between disruptions (say $T_{1}$ ), the performance loss $X$ and the time needed for recovery (say $T_{2}$ ) (see ${ }^{36}$ and ${ }^{37}$ ). In this context, the idea would be to develop new TBEA type control charts for which the time is no longer a univariate random variable but a bivariate one $\mathbf{T}=\left(T_{1}, T_{2}\right)$ and the amplitude $X$ remains the same as for traditional TBEA type data.

Similar statistics to the ones used in this paper can also be investigated in the case where time between events $T$ is a univariate random variable and the amplitude is no longer a univariate random variable $X$ but is a multivariate random vector $\mathbf{X}$ (containing the different types of amplitude corresponding to a particular kind of negative event). In addition, other continuous distributions like the Beta or Rayleigh distributions (as in Ali and Riaz ${ }^{38}$ for instance), as well as discrete distributions for the amplitude $X$ like the Binomial or Poisson distribution could also be considered.

\section{Authors bio}

Dorra Rahali is a PhD student at the Universite de Nantes and the Université de Tunis. She is a member of the LS2N (Laboratoire des Sciences du Numérique de Nantes) UMR CNRS 6004 and the ARBRE Laboratory (Applied Research in Business Relationships and Economics). She received her MS in Production from the higher school of sciences and techniques, Université de Tunis in 2012. Her research interests include Statistical Quality Control methods.

Philippe Castagliola is graduated (PhD 1991) from the UTC (Université de Technologie de Compiègne, France). He is currently full professor at the Université de Nantes, Nantes, France, and he is also a member of the LS2N (Laboratoire des Sciences du Numérique de Nantes), UMR CNRS 6004. He is an associate editor for Quality Engineering, Communications in Statistics (LSTA, LSSP, UCAS) and Quality Technology \& Quantitative Management. His research activity includes developments of new Statistical Process Monitor- 

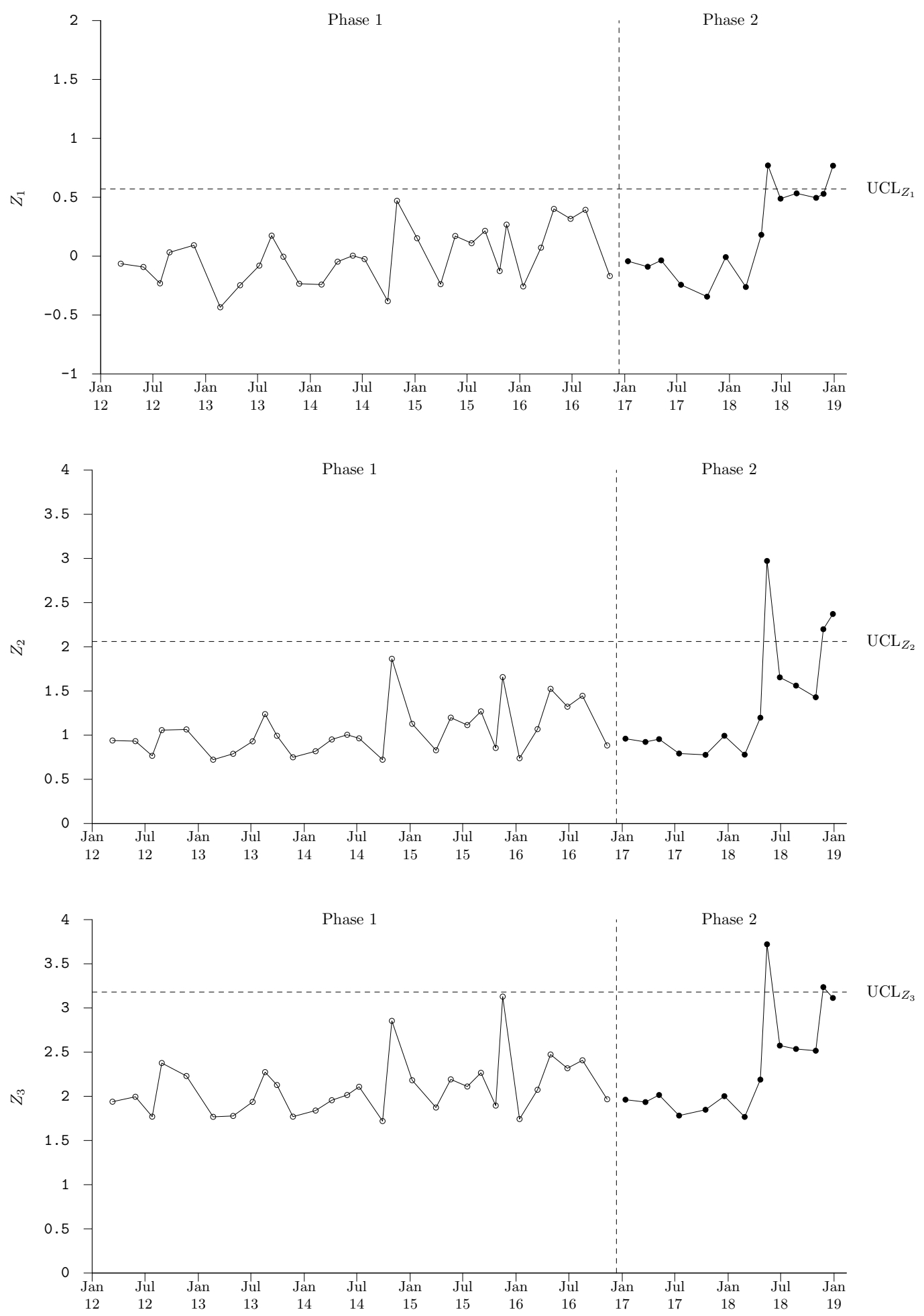

Figure 4: Statistics $Z_{1}, Z_{2}$ and $Z_{3}$ corresponding to the data set in Table 11. 
ing techniques.

Hassen Taleb is a professor of statistics at the University of Carthage. He earned a PhD of statistics from University of Tunis. He is member of ARBRE Laboratory at the Higher Institute of Management. Research interest involves statistical process control and includes monitoring attribute process.

Michael Boon Chong Khoo is a professor in the School of Mathematical Sciences, Universiti Sains Malaysia (USM). He received his PhD in Applied Statistics in 2001 from USM. His research interest is in statistical process control. $\mathrm{He}$ is a member of the American Society for Quality.

\section{References}

[1] Z. Wu, J. Jiao, and Z. He. A Single Control Chart for Monitoring the Frequency and Magnitude of an Event. International Journal of Production Economics, 119(1):24-33, 2009.

[2] Y. Cheng, A. Mukherjee, and M. Xie. Simultaneously Monitoring Frequency and Magnitude of Events Based on Bivariate Gamma Distribution. Journal of Statistical Computation and Simulation, 87(9):1723-1741, 2017.

[3] T. Calvin. Quality Control Techniques for "Zero Defects". IEEE Transactions on Components, Hybrids, and Manufacturing Technology, 6(3):323$328,1983$.

[4] J.M. Lucas. Counted Data CUSUM's. Technometrics, 27(2):129-144, 1985.

[5] S. Vardeman and D.O. Ray. Average Run Lengths for CUSUM Schemes when Observations are Exponentially Distributed. Technometrics, 27(2): 145-150, 1985.

[6] L.Y. Chan, M. Xie, and T.N. Goh. Cumulative Quantity Control Charts for Monitoring Production Processes. International Journal of Production Research, 38(2):397-408, 2000. doi: 10.1080/002075400189482.

[7] M. Xie, T.N. Goh, and P. Ranjan. Some Effective Control Chart Procedures for Reliability Monitoring. Reliability Engineering \& System Safety, 77(2): 143-150, 2002.

[8] P.D. Bourke. The Geometric CUSUM Chart with Sampling Inspection for Monitoring Fraction Defective. Journal of Applied Statistics, 28(8): 951-972, 2001.

[9] F.F. Gan. Exact Run Length Distributions for One-Sided Exponential CUSUM Schemes. Statistica Sinica, 2(1):297-312, 1992.

[10] C.M. Borror, J.B. Keats, and D.C. Montgomery. Robustness of the Time Between Events CUSUM. International Journal of Production Research, 41(15):3435-3444, 2003. 
[11] M.S. Shafae, R.M. Dickinson, W.H. Woodall, and J.A. Camelio. Cumulative Sum Control Charts for Monitoring Weibull-Distributed Time Between Events. Quality and Reliability Engineering International, 31(5):839-849, 2015.

[12] F.F. Gan. Designs of One- and Two-Sided Exponential EWMA Charts. Journal of Quality Technology, 30(1):55-69, 1998.

[13] J.Y. Liu, M. Xie, and T.N. Goh. CUSUM Chart with Transformed Exponential Data. Communications in Statistics-Theory and Methods, 35(10): 1829-1843, 2006.

[14] C.W. Zhang, M. Xie, J.Y. Liu, and T.N. Goh. A Control Chart for the Gamma Distribution as a Model of Time Between Events. International Journal of Production Research, 45(23):5649-5666, 2007.

[15] Y.J. Xie, K.L. Tsui, M. Xie, and T.N. Goh. Monitoring Time-BetweenEvents for Health Management. In Prognostics and Health Management Conference, 2010. PHM10., pages 1-8. IEEE, 2010.

[16] Y.Y. Fang, M.B.C. Khoo, S.Y. Teh, and M. Xie. Monitoring of TimeBetween-Events with a Generalized Group Runs Control Chart. Quality and Reliability Engineering International, 32(3):767-781, 2016.

[17] Z. Wu, J. Jiao, and H. Zhen. A Control Scheme for Monitoring the Frequency and Magnitude of an Event. International Journal of Production Research, 47(11):2887-2902, 2009.

[18] Z. Wu and L. Qu. A Single Chart for Monitoring Frequency and Magnitude of Events. In Industrial Engineering and Engineering Management (IEEM), 2010 IEEE International Conference on, pages 1416-1420. IEEE, 2010.

[19] L. Qu, Z. Wu, M.B.C. Khoo, and P. Castagliola. A CUSUM Scheme for Event Monitoring. International Journal of Production Economics, 145(1): 268-280, 2013.

[20] L. Qu, S. He, M.B.C. Khoo, and P. Castagliola. A CUSUM Chart for Detecting the Intensity Ratio of Negative Events. International Journal of Production Research, 56(19):6553-6567, 2018.

[21] S. Ali and A. Pievatolo. Time and Magnitude Monitoring Based on the Renewal Reward Process. Reliability Engineering \&6 System Safety, 179: 97-107, 2018.

[22] Y. Cheng and A. Mukherjee. One Hotelling $T^{2}$ Chart Based on Transformed Data for Simultaneous Monitoring the Frequency and Magnitude of an Event. In 2014 IEEE International Conference on Industrial Engineering and Engineering Management, pages 764-768, Dec 2014. doi: 10.1109/IEEM.2014.7058741.

[23] R.A. Sanusi, S.Y. Teh, and M.B.C. Khoo. Simultaneous Monitoring of Magnitude and Time-Between-Events Data with a Max-EWMA Control Chart. Computers \& Industrial Engineering, 142:106378, 2020. doi: 10.1016/j.cie.2020.106378. 
[24] M. Sklar. Fonctions de Répartition à $n$ Dimensions et leurs Marges. Publ. inst. statist. univ. Paris, 8:229-231, 1959.

[25] A.A. Fatahi, P. Dokouhak, and B.F. Moghaddam. A Bivariate Control Chart Based on Copula Function. In Quality and Reliability (ICQR), 2011 IEEE International Conference on, pages 292-296. IEEE, 2011.

[26] P. Dokouhaki and R. Noorossana. A Copula Markov CUSUM Chart for Monitoring the Bivariate Auto-Correlated Binary Observations. Quality and reliability engineering international, 29(6):911-919, 2013.

[27] P. Busababodhin and P. Amphanthong. Copula Modelling for Multivariate Statistical Process Control: A Review. Communications for Statistical Applications and Methods, 23(6):497-515, 2016.

[28] S. Sukparungsee, S. Kuvattana, P. Busababodhin, and Y. Areepong. Bivariate Copulas on the Hotelling's $T^{2}$ Control Chart. Communications in Statistics-Simulation and Computation, 47(2):413-419, 2018.

[29] D. Rahali, P. Castagliola, H. Taleb, and M.B.C. Khoo. Evaluation of Shewhart Time-Between-Events-and-Amplitude Control Charts for Several Distributions. Quality Engineering, 2019. doi: 10.1080/08982112.2018.1479036.

[30] M.C. Wang, J. Qin, and C.T. Chiang. Analyzing Recurrent Event Data with Informative Censoring. Journal of the American Statistical Association, 96(455):1057-1065, 2001.

[31] C.Y. Huang and M.C. Wang. Joint Modeling and Estimation for Recurrent Event Processes and Failure Time Data. Journal of the American Statistical Association, 99(468):1153-1165, 2004.

[32] V. Rondeau. Statistical Models for Recurrent Events and Death: Application to Cancer Events. Mathematical and Computer Modelling, 52(7-8): 949-955, 2010.

[33] M.J. Frank. On the Simultaneous Associativity of $F(x, y)$ and $x+y-F(x, y)$. Aequationes mathematicae, 19(1):194-226, 1979.

[34] D.G. Clayton. A Model for Association in Bivariate Life Tables and its Application in Epidemiological Studies of Familial Tendency in Chronic Disease Incidence. Biometrika, 65(1):141-151, 1978.

[35] E.J. Gumbel. Distributions Des Valeurs Extremes en Plusieurs Dimensions. Publ. Inst. Statist. Univ. Paris, 9:171-173, 1960.

[36] L. Shen, B. Cassottana, and L.C. Tang. Statistical Trend Tests for Resilience of Power Systems. Reliability Engineering 83 System Safety, 177: 138-147, 2018.

[37] B. Cassottana, L. Shen, and L.C. Tang. Modeling the Recovery Process: A Key Dimension of Resilience. Reliability Engineering 83 System Safety, 190:106528, 2019.

[38] S. Ali and M. Riaz. Cumulative Quantity Control Chart for the Mixture of Inverse Rayleigh Process. Computers \& Industrial Engineering, 73:11-20, 2014. 